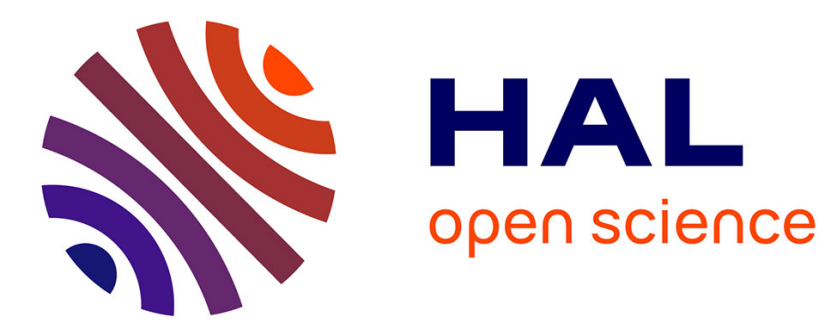

\title{
Existential Rules: A Graph-Based View
}

Marie-Laure Mugnier

\section{To cite this version:}

Marie-Laure Mugnier. Existential Rules: A Graph-Based View. Pablo Barceló; Reinhard Pichler. Datalog 2.0, Sep 2012, Vienne, Austria. Springer, 2nd International Workshop on Datalog 2.0, LNCS (7494), pp.21-26, 2012, 10.1007/978-3-642-32925-8_3. lirmm-00763474

\section{HAL Id: lirmm-00763474 https://hal-lirmm.ccsd.cnrs.fr/lirmm-00763474}

Submitted on 16 Sep 2019

HAL is a multi-disciplinary open access archive for the deposit and dissemination of scientific research documents, whether they are published or not. The documents may come from teaching and research institutions in France or abroad, or from public or private research centers.
L'archive ouverte pluridisciplinaire HAL, est destinée au dépôt et à la diffusion de documents scientifiques de niveau recherche, publiés ou non, émanant des établissements d'enseignement et de recherche français ou étrangers, des laboratoires publics ou privés. 


\section{Existential Rules:}

\section{A Graph-based View}

Marie-Laure Mugnier

University of Montpellier 


\section{Ontology-based Data Access (OBDA)}

\section{Answers ?}

\section{Knowledge Base}

Data

Adding an ontological layer:

to abstract from a specific database schema

to provide a unified view of mutiple sources

to infer new facts, thus allowing for data incompleteness 


\section{Outline}

Existential rules: a logic- and graph-based framework

Decidability and algorithmic issues

- Focus on:

- tree-shaped saturation in forward chaining

- piece-based unification in backward chaining

A (graph) tool for combining decidable classes of rules 


\section{Data / Facts}

Relational Database

$$
\begin{aligned}
& \text { parentOf Male Fem. } \\
& a \mathrm{~b} \\
& \text { a } c \\
& \text { c ? }
\end{aligned}
$$

RDF (Semantic Web)

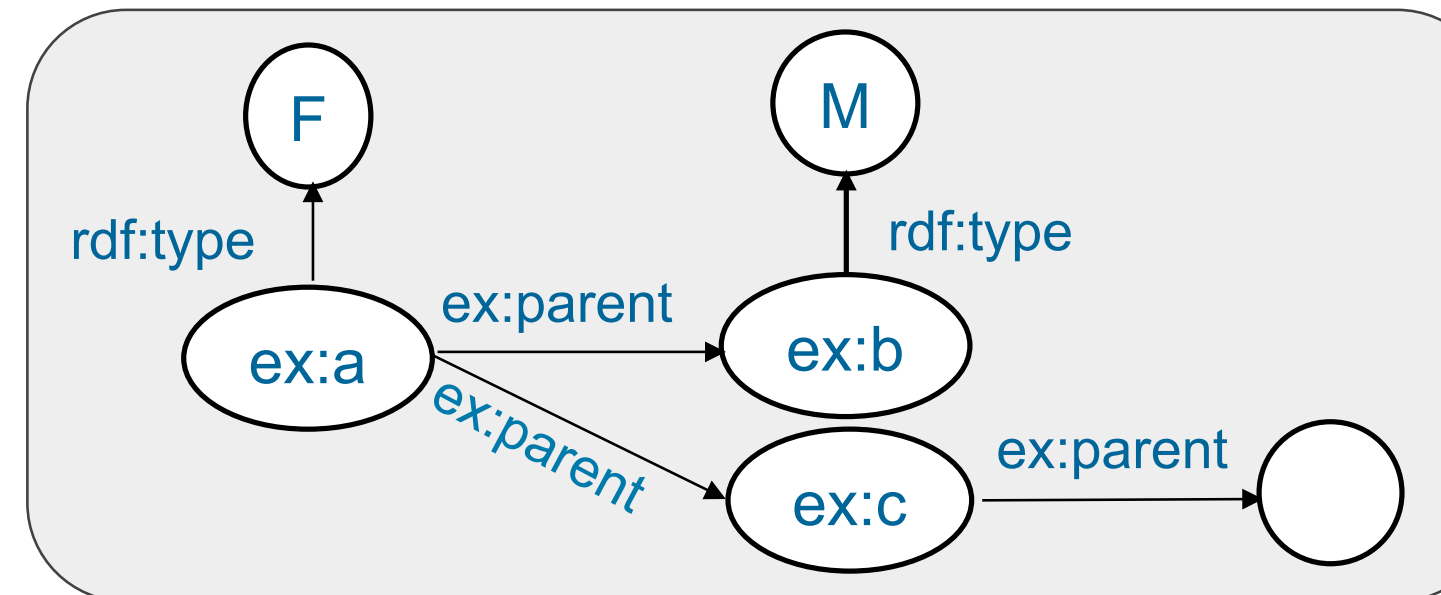

Etc.

Abstraction in first-order logic

$\exists x($ parentOf $(a, b) \wedge$ parentOf $(a, c) \wedge$ parentOf $(c, x) \wedge F(a) \wedge M(b))$
Or in graphs / hypergraphs

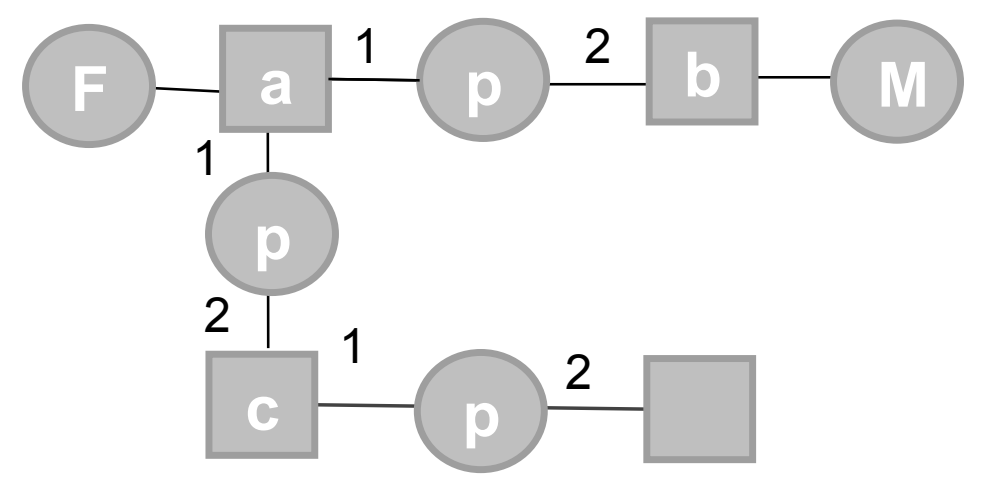




\section{Ontology: Existential Rules}

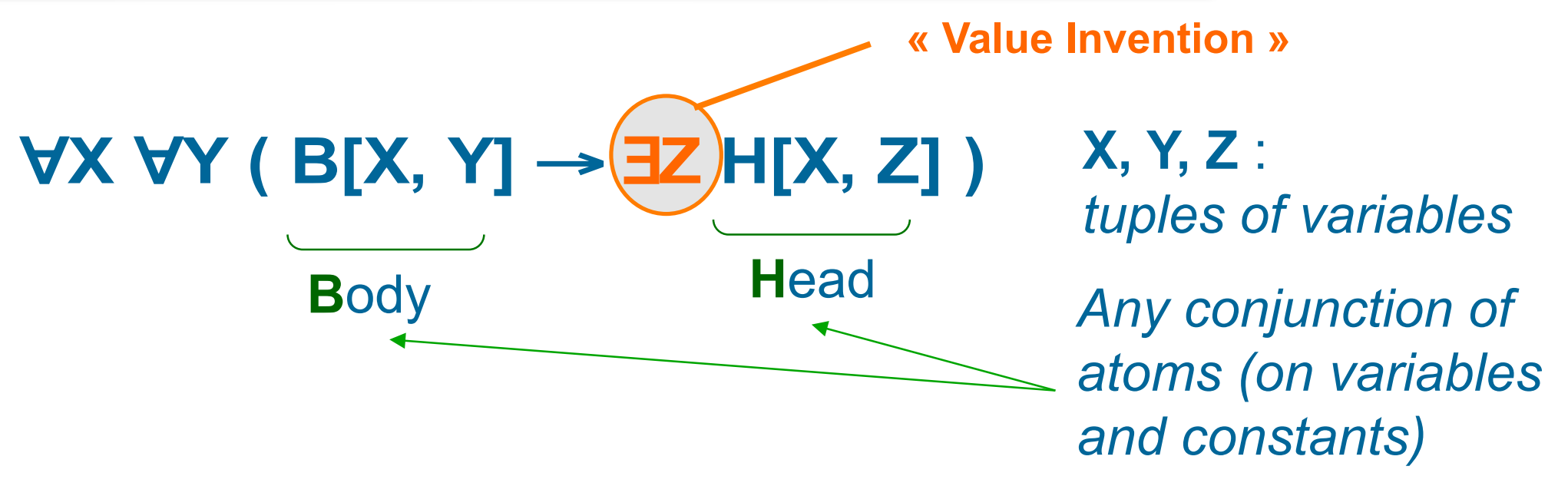

$\forall x \forall y($ siblingOf $(x, y) \rightarrow \exists z($ parentOf $(z, x) \wedge$ parentOf $(z, y)))$

Simplified form: siblingOf $(x, y) \rightarrow$ parentOf $(z, x) \wedge$ parentOf $(z, y)$

- Same as Tuple Generating Dependencies (TGDs)

- See also Datalog+/-

- Same as the logical translation of Conceptual Graph rules

- Generalize Description Logics used for OBDA (DL-Lite, $\mathcal{E L}$ ) 


\section{Ontology: Existential Rules}

\section{$\forall X \forall Y(B[X, Y] \rightarrow \exists Z H[X, Z])$ graph graph}

$\forall x \forall y($ siblingOf $(x, y) \rightarrow \exists z($ parentOf $(z, x) \wedge$ parentOf $(z, y)))$

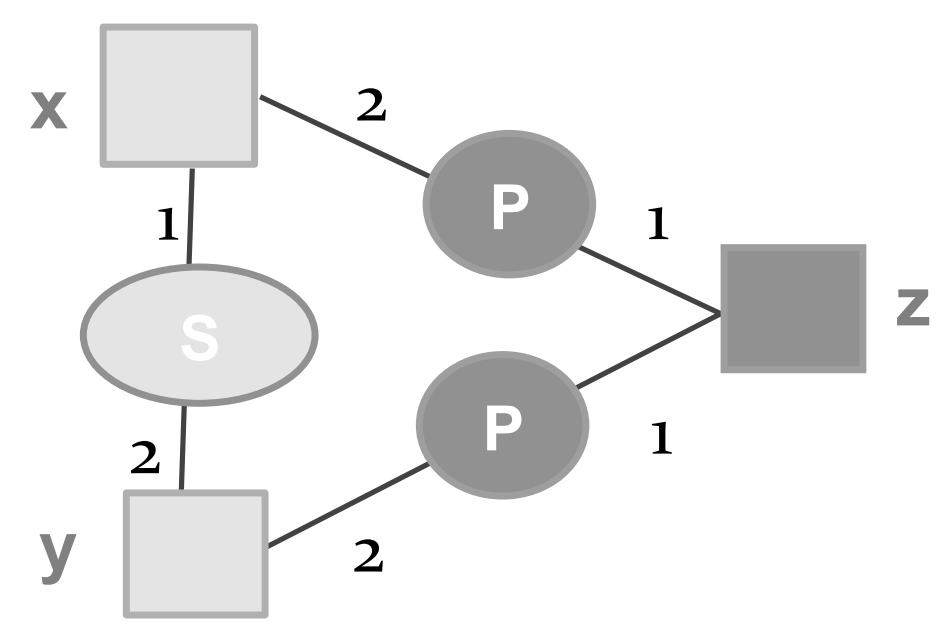




\section{Value Invention}

$R=\forall x \forall y($ siblingOf $(x, y) \rightarrow \exists z($ parentOf $(z, x) \wedge$ parentOf $(z, y)))$

$\mathrm{F}=$ siblingOf $(a, b)$
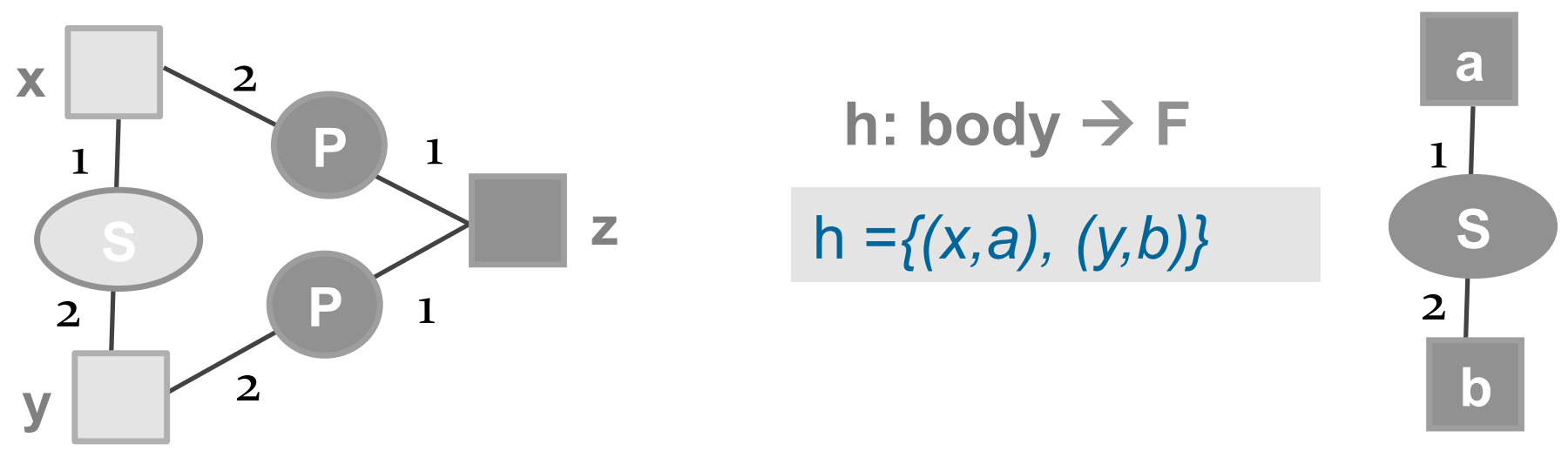

A rule body $\rightarrow$ head is applicable to a fact $F$ if there is a homomorphism $h$ : body $\rightarrow F$

Then $h$ (head) can be " added " to $F$ with renaming existential variables of head $F^{\prime}=\exists$ z0 (siblingOf(a,b)

^ parentOf $(\mathrm{ZO}, \mathrm{a}) \wedge$ parentOf( $(\mathrm{ZO}, \mathrm{a}))$

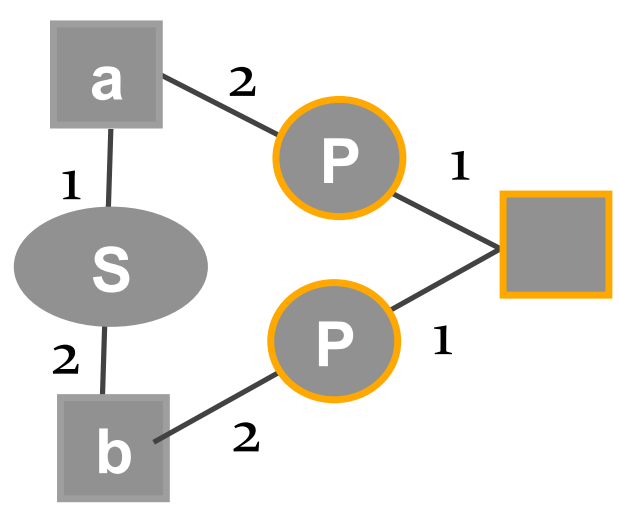




\section{Logical /Graphical Framework}

\section{Answers ?}

Knowledge Base
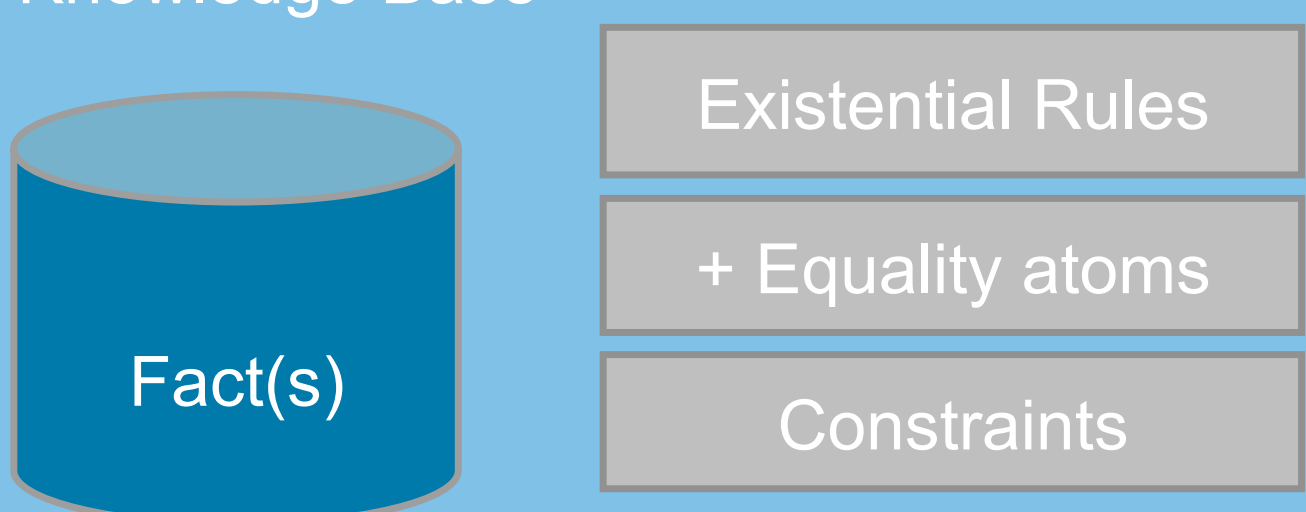

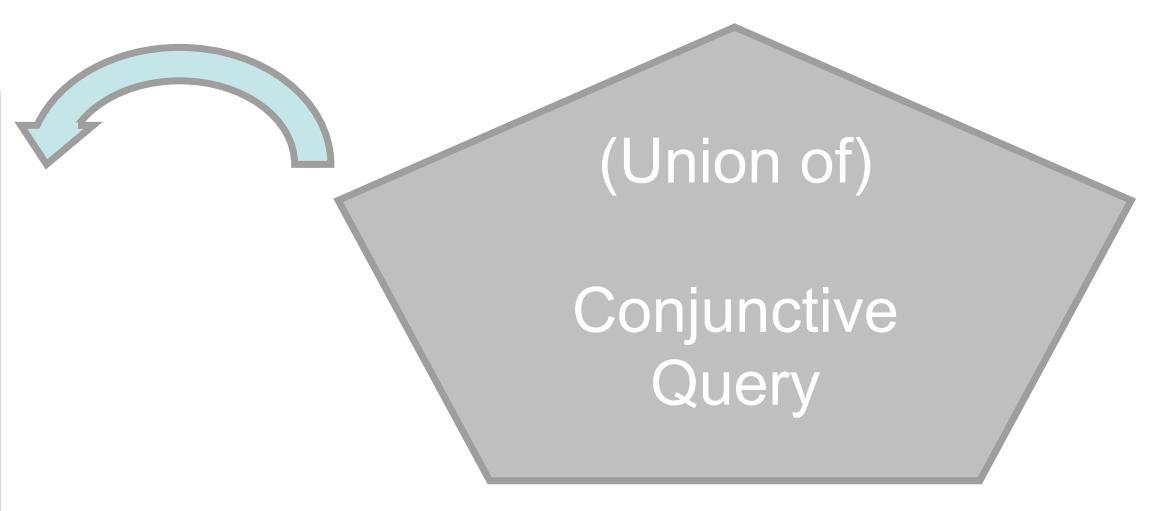

(V) $\exists X F[X]$

Negative constraint:

$\neg(\exists \mathrm{X} B[\mathrm{X}])$ or $\forall \mathrm{X}(\mathrm{B}[\mathrm{X}] \rightarrow \perp)$

« $B[X]$ must not be found »

Positive constraint:

$$
\forall X \forall Y(B[X, Y] \rightarrow \exists Z H[X, Z])
$$

" if $B[X, Y]$ is found then $H[X, Z]$ must also be found " 


\section{Similar Framework: Datalog +/-}

\section{Answers ?}

\section{Knowledge Base}
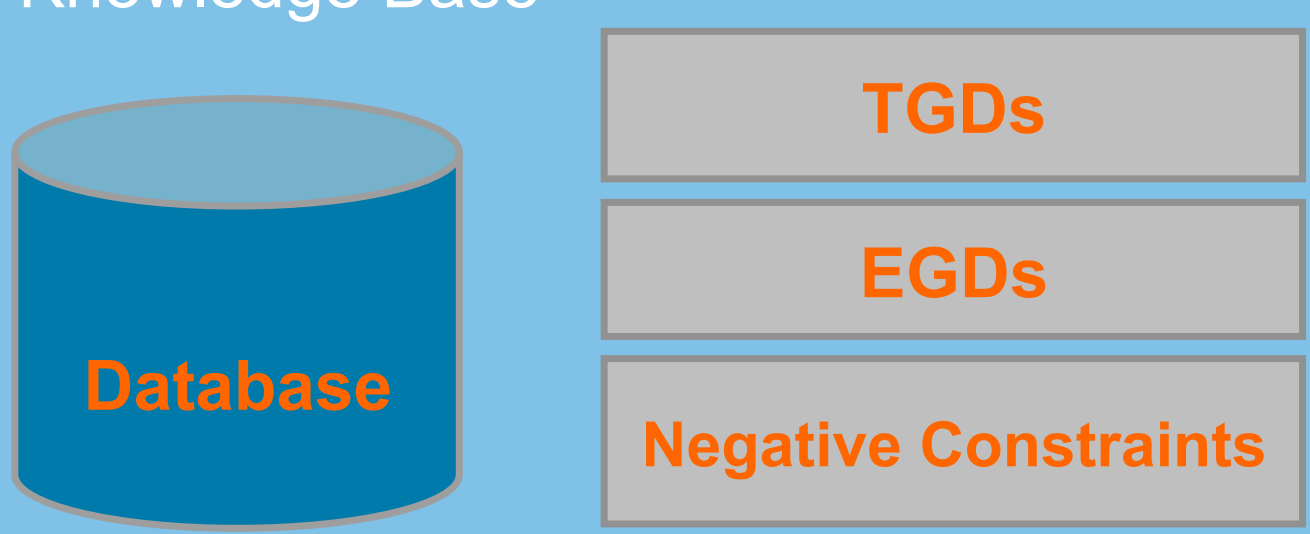

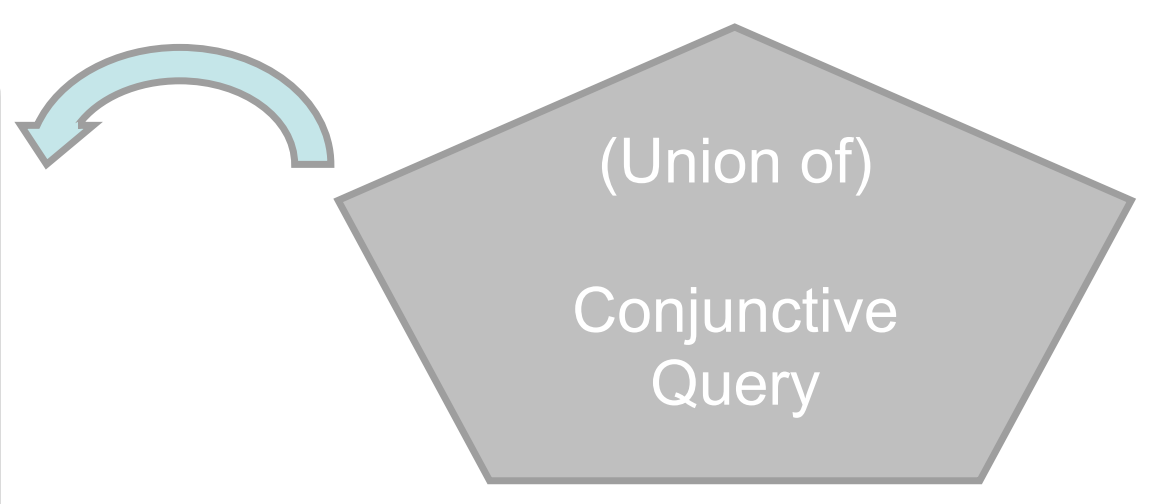

[Cali Gottlob Lukasiewicz PODS 2009]

Tuple Generating Dependency $=($ pure $)$ existential rule

Equality Generating Dependency: $\forall X(B[X] \rightarrow x=e)$ 


\section{The Conceptual Graph Origins}

Conceptual graphs introduced in [Sowa 76] [Sowa 84]

- Specific research line by Montpellier's group since 1992

« Graph-based » knowledge representation and reasoning

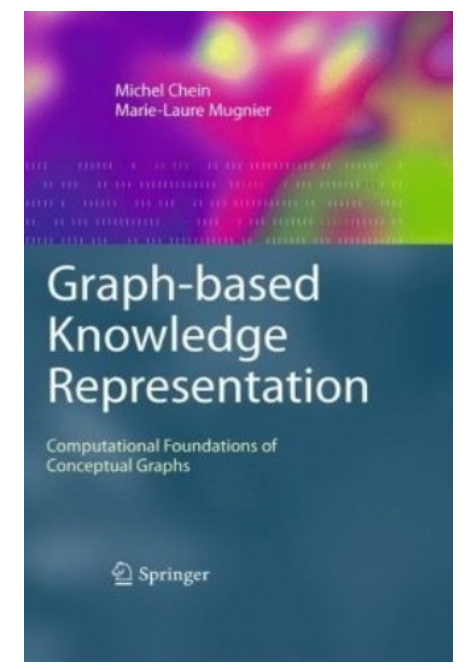

" Graph-Based Knowledge Representation: Computational Foundations of Conceptual Graphs », Chein \& M..., Springer, 2009 


\section{Conceptual Graph Vocabulary:}

1. partially (pre-)ordered set of concepts
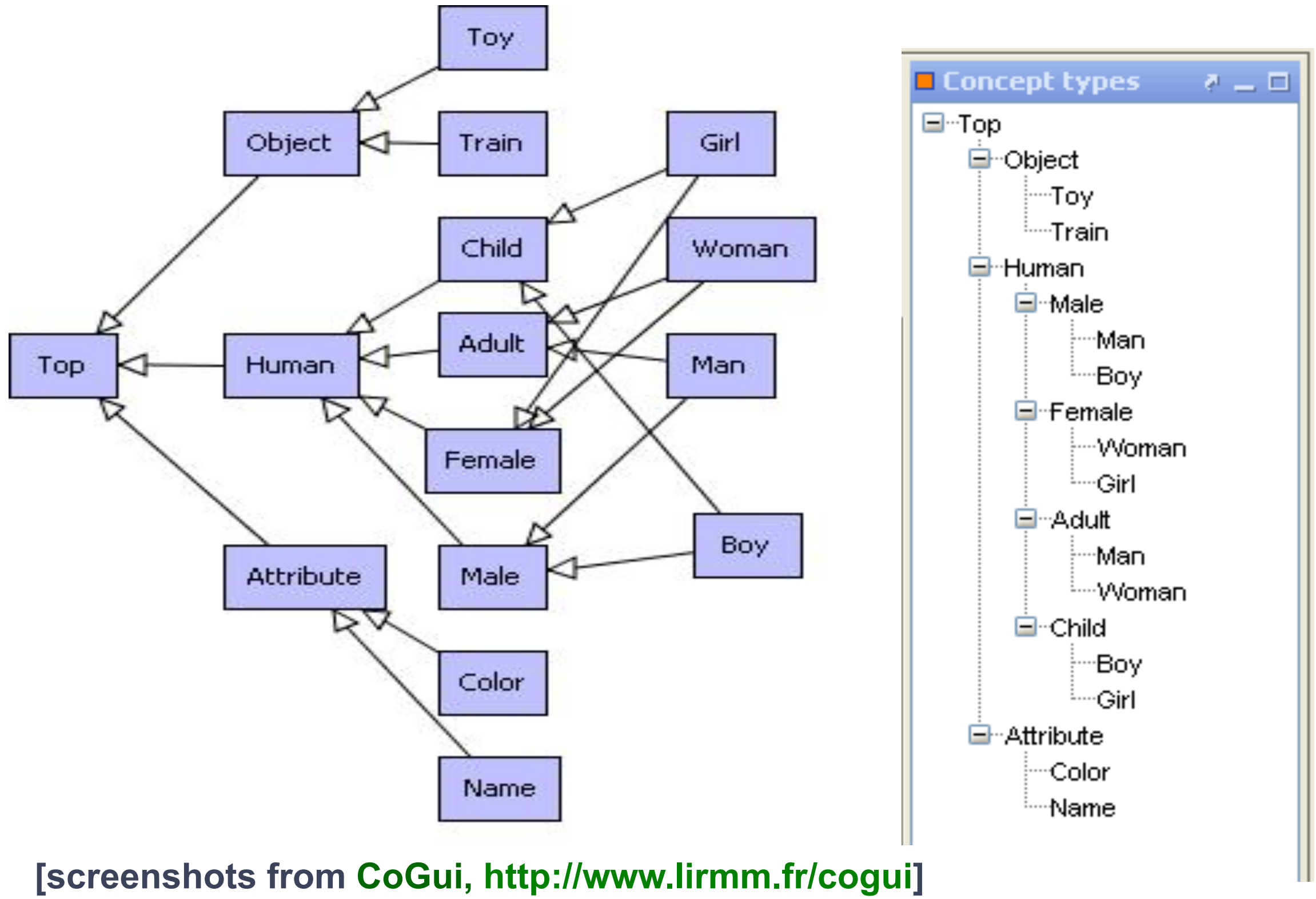

[screenshots from CoGui, http://www.lirmm.fr/cogui] 
Conceptual Graph Vocabulary:

2. partially (pre-)ordered set of relations with their signature [any relation arity allowed]
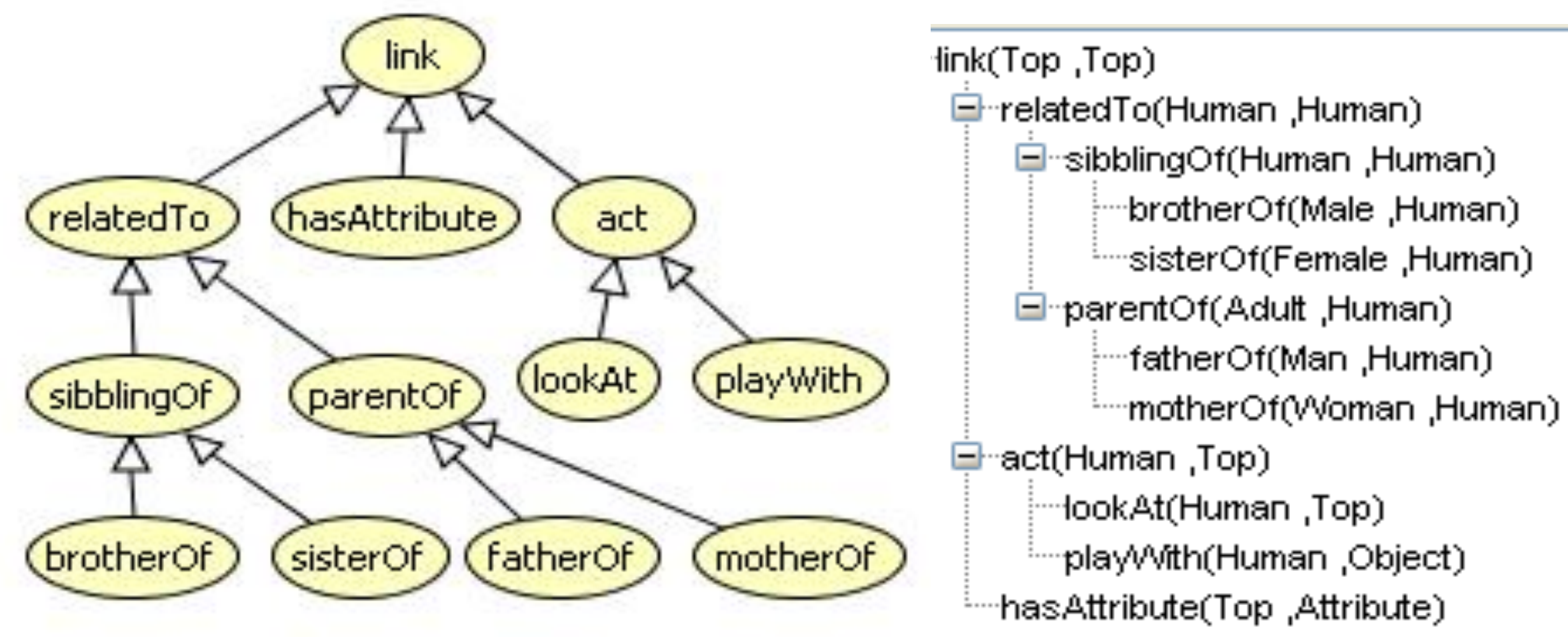

Logical translation $(\Phi)$ of the vocabulary: very simple rules

$p<q \quad \forall x_{1} \ldots x_{k}\left(p\left(x_{1} \ldots x_{k}\right) \rightarrow q\left(x_{1} \ldots x_{k}\right)\right)$

Signature of $r \quad \forall x_{1} \ldots x_{k}\left(p\left(x_{1} \ldots x_{k}\right) \rightarrow t_{i 1}\left(x_{1}\right) \ldots t_{i k}\left(x_{k}\right)\right)$ 


\section{Basic Conceptual Graph}

Eva

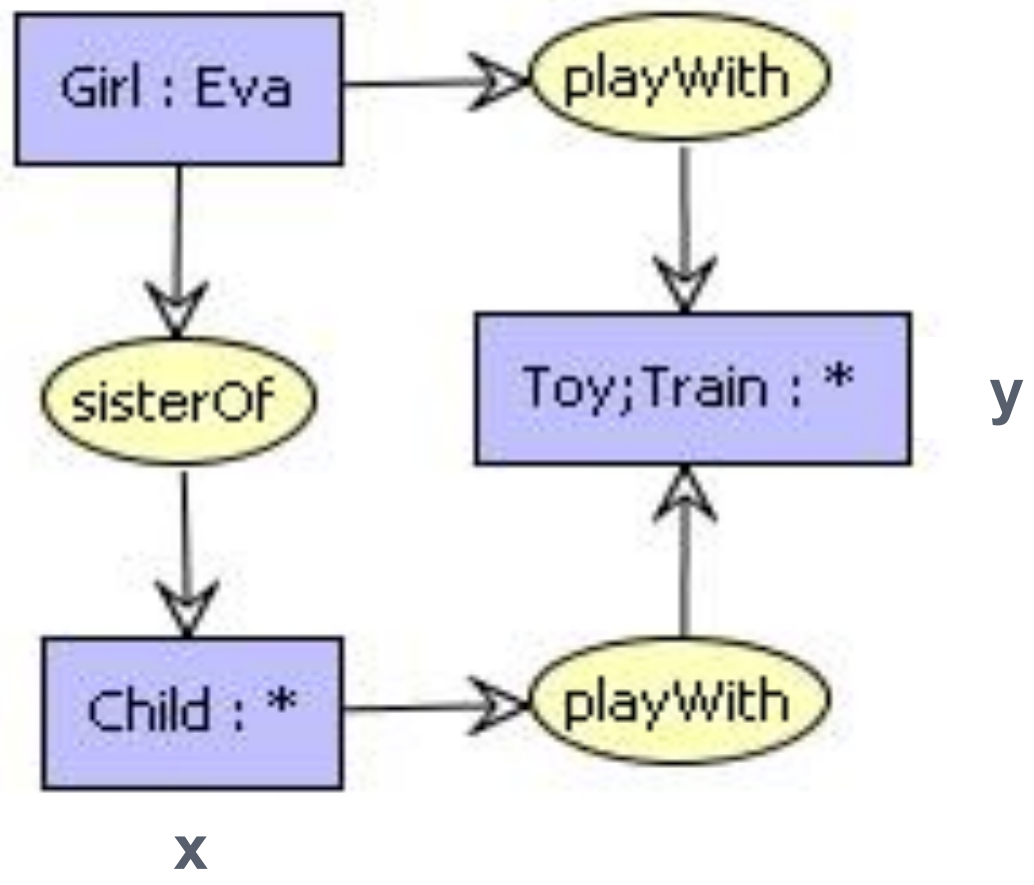

[total order on the edges incident to a relation node]

Logical translation $(\Phi)$ : existentially closed conjunction of atoms

$\exists x \exists y($ Girl(Eva) $\wedge$ Child $(x) \wedge \operatorname{Toy}(y) \wedge \operatorname{Train}(y)$

$\wedge$ sisterOf $(E v a, x) \wedge$ playWith $(E v a, y) \wedge$ playWith $(x, y))$

Allows to represent facts and conjunctive queries 
Homomorphism (with concept/relation preorders integrated)

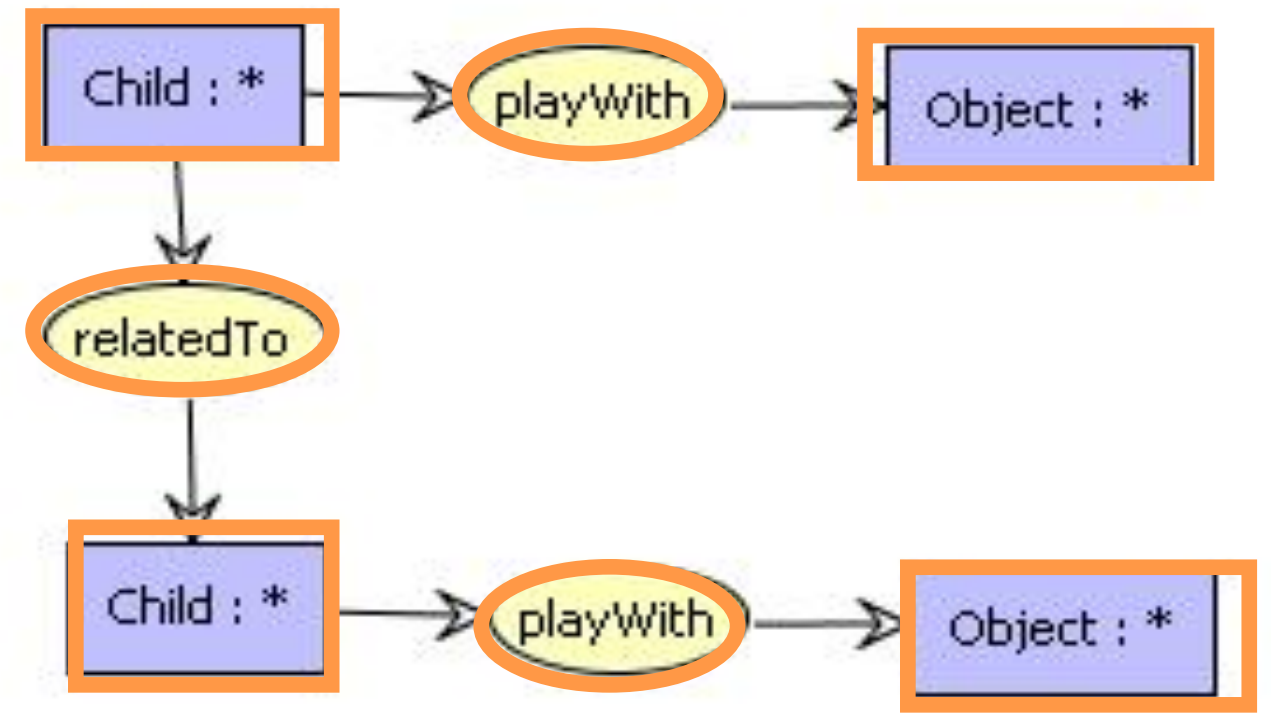

\section{Fact $\boldsymbol{F}$}

Query Q

Logical soundness [Sowa 84] and completeness

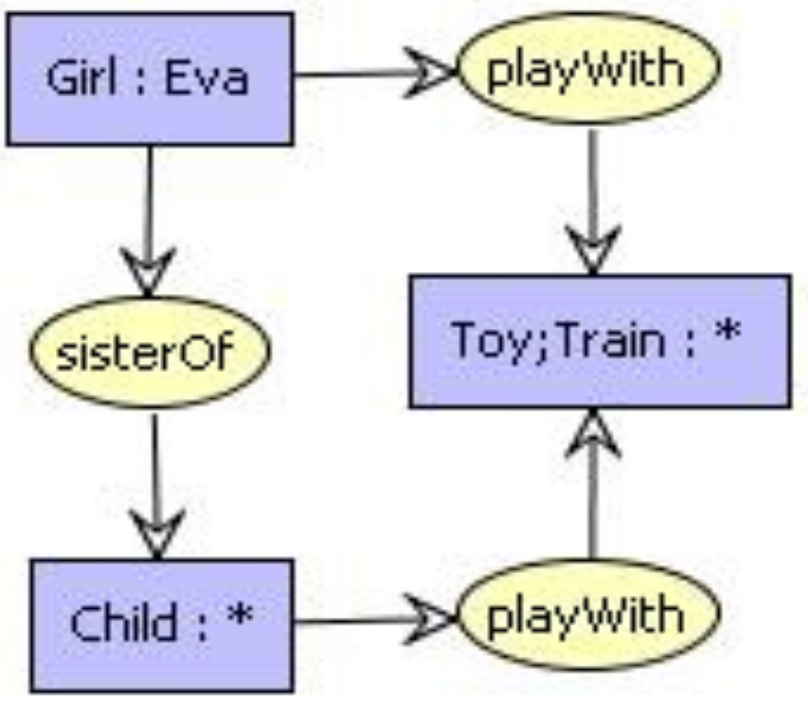
[Chein M... 92]:

there is a homomorphism from $Q$ to $F$ iff $\Phi(Q)$ is entailed by $\Phi(F)$ and $\Phi($ vocabulary)

The Basic CG fragment restricted to binary relations is equivalent to RDFS [Baget ISWC' 05] [Baget+ ICCS' 10] 
Richer Fragments (nested graphs, rules, constraints, + negation, ...)

- Rule: pair of basic conceptual graphs

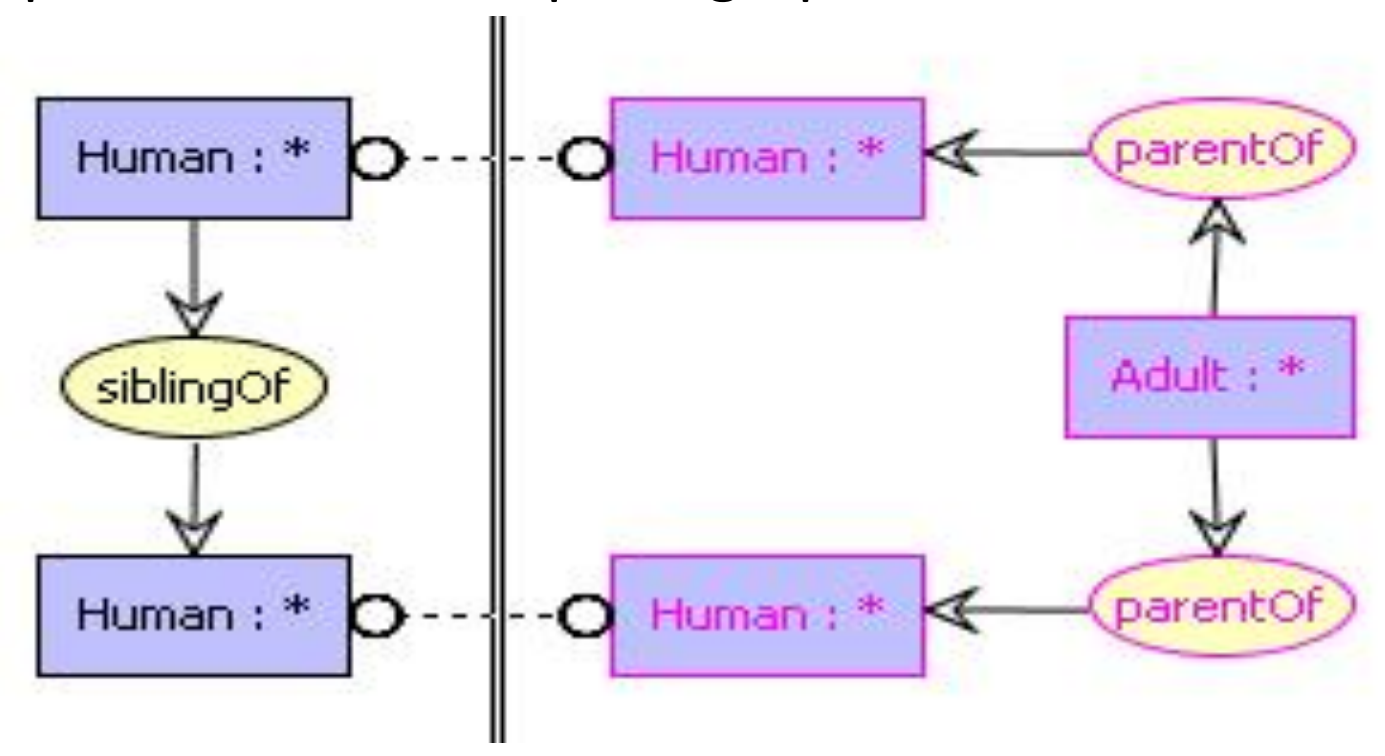

$\forall x \forall y(\operatorname{Human}(x) \wedge \operatorname{Human}(\mathrm{y}) \wedge$ siblingOf $(\mathrm{x}, \mathrm{y})$

$\rightarrow \exists z(\operatorname{Adult}(z) \wedge \operatorname{parentOf}(z, x) \wedge \operatorname{parentOf}(z, y)))$

- Sound and complete forward chaining and backward chaining [Salvat M... 1996]

- Several ways of combining rules and constraints [Baget M... JAIR 2002]

The existential rule framework can be seen as a fragment of CGs with a flat vocabulary 


\section{Outline}

Existential rules: a logic- and graph-based framework

Decidability and algorithmic issues

- Focus on:

tree-shaped saturation in forward chaining piece-based unification in backward chaining

A (graph) tool for combining decidable classes of rules 


\section{Basic Problem}

\section{Knowledge Base}

\section{Answers ?}
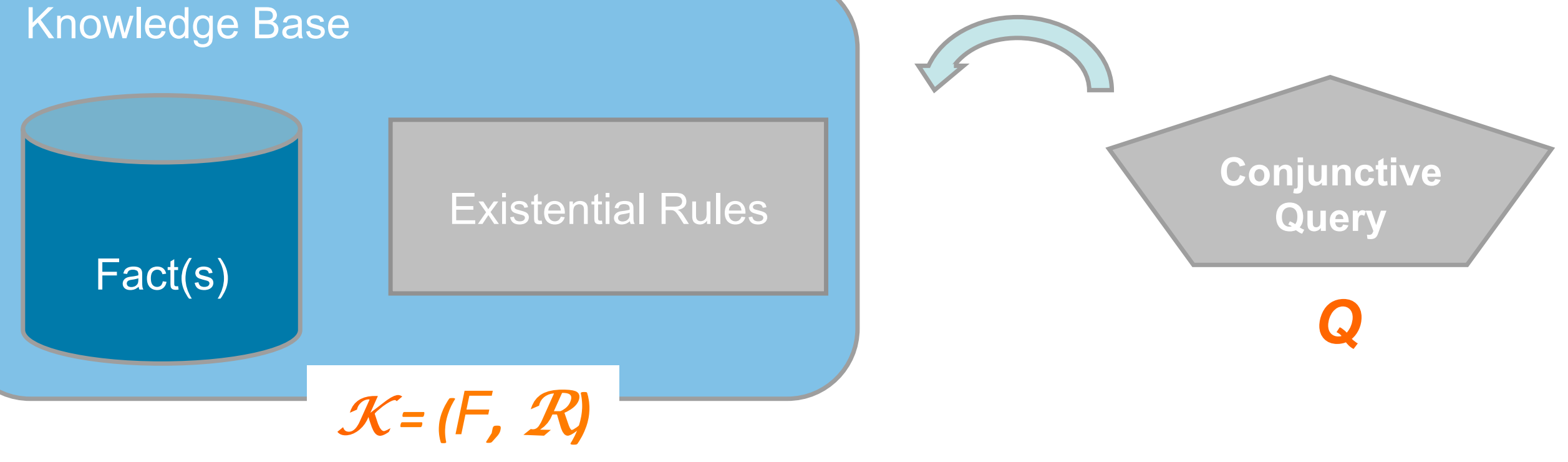

Conjunctive Query Entailment

Given a KB $\mathcal{K}=(\boldsymbol{F}, \mathcal{R})$ and a (Boolean) conjunctive query $\boldsymbol{Q}$, is $Q$ entailed by $\mathcal{K}$ ? 


\section{Forward vs Backward Chaining}

FC Fact saturation (" chase », " bottom-up »)

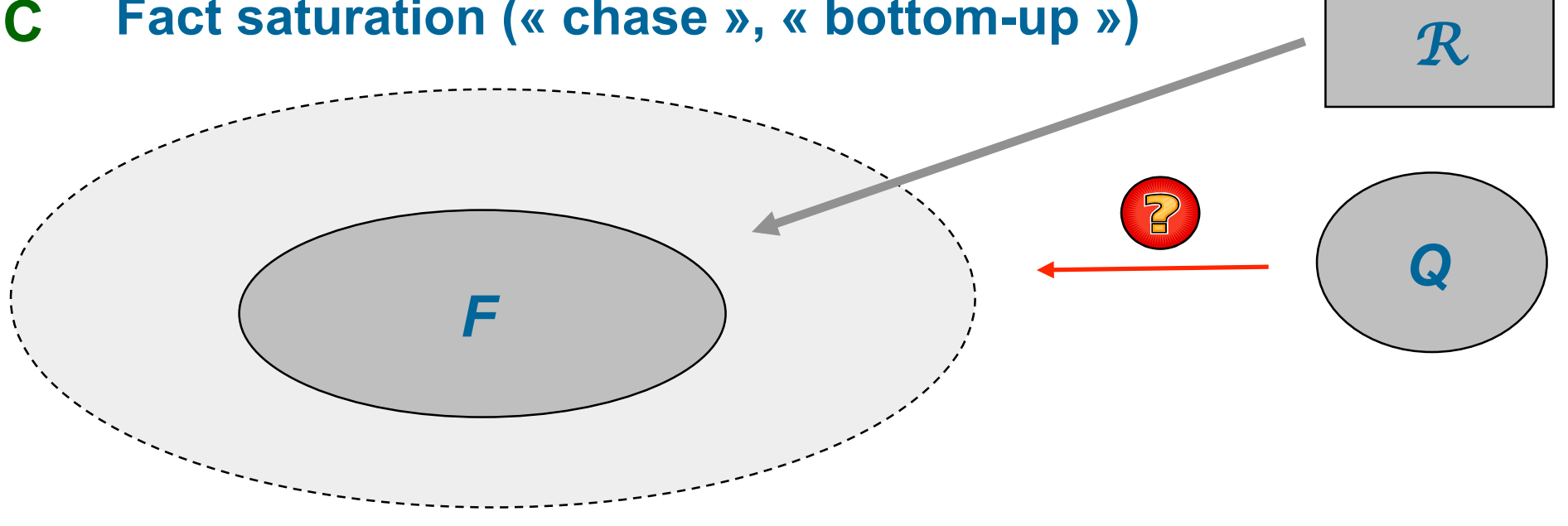

BC Query rewriting («top-down »)

[Decomposition into 2 steps: DL-Lite]
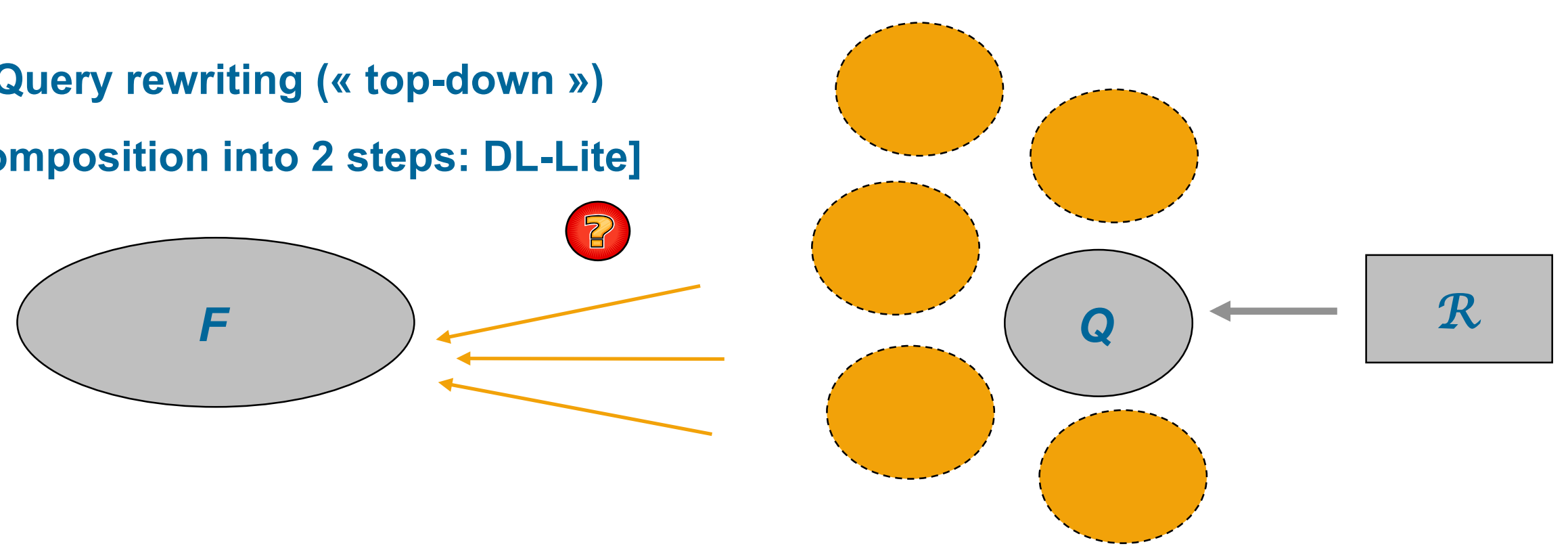


\section{Decidability Issues}

Entailment is not decidable

Many decidable classes exhibited in databases and KR

Three generic kinds of properties ensuring decidability:

- Saturation by Forward Chaining halts (« finite expansion set », fes)

- Query rewriting by Backward Chaining halts (" finite unification set », fus)

- Saturation by Forward Chaining may not halt but the generated facts have a tree-like structure (" bounded treewidth set », bts)

None of these properties is recognizable [Baget+ KR 10] but they provide generic algorithms 


\section{Decomposition Tree / Treewidth}
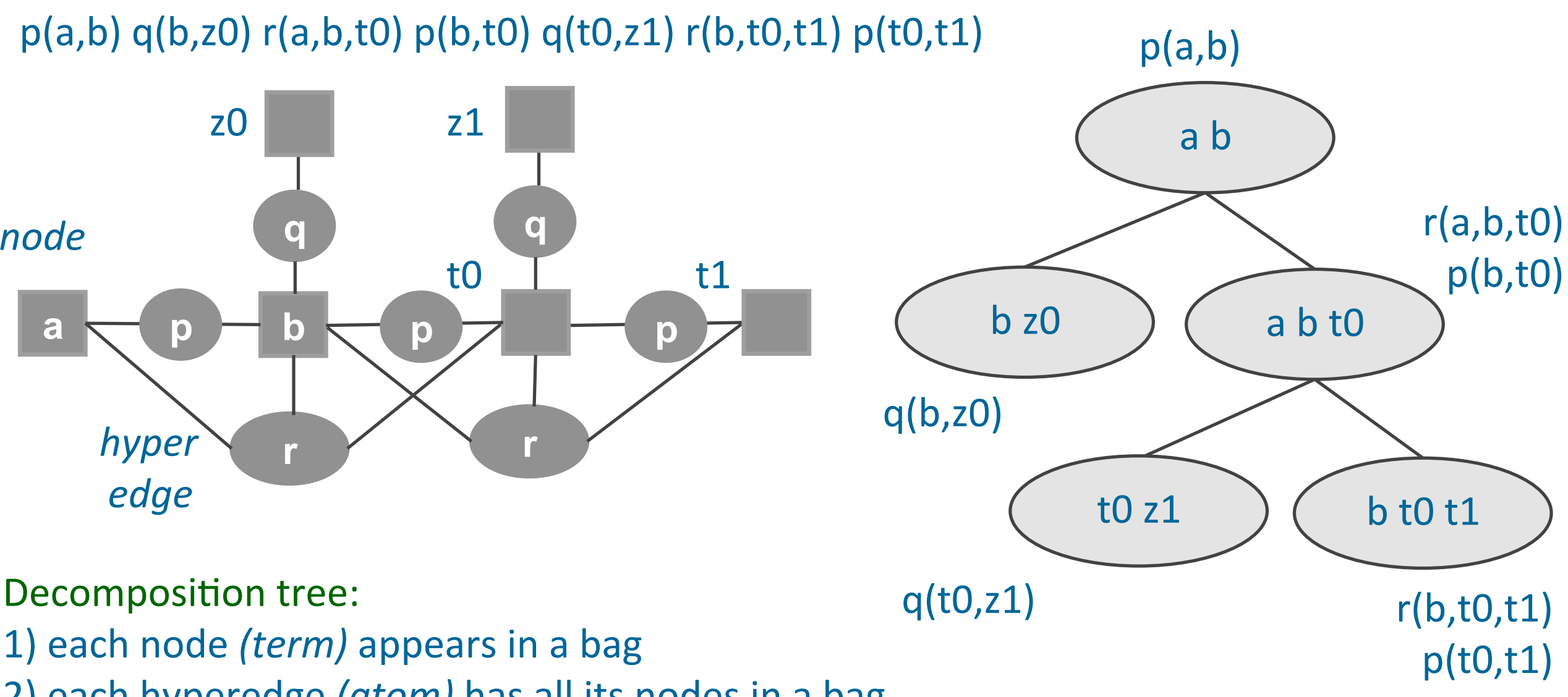

Decomposition tree:

1) each node (term) appears in a bag

2) each hyperedge (atom) has all its nodes in a bag

3) for each node $x$, the subgraph induced by the bags containing $x$ is connected

Width of a tree decomposition = max number of nodes in a bag (minus 1 )

Treewidth of a graph $=$ min width over all decomposition trees of this graph 


\section{Bounded Treewidth of the Derived Facts (bts)}

Essentially [Cali Gottlob Kifer KR'08]

$\mathcal{R}$ is bts if FC with $\mathcal{R}$ generates facts with bounded treewidth

i.e., for any fact $F$, there is an integer $b$ s.t.

any fact $\mathcal{R}$-derived from $F$ has treewidth bounded by $b$

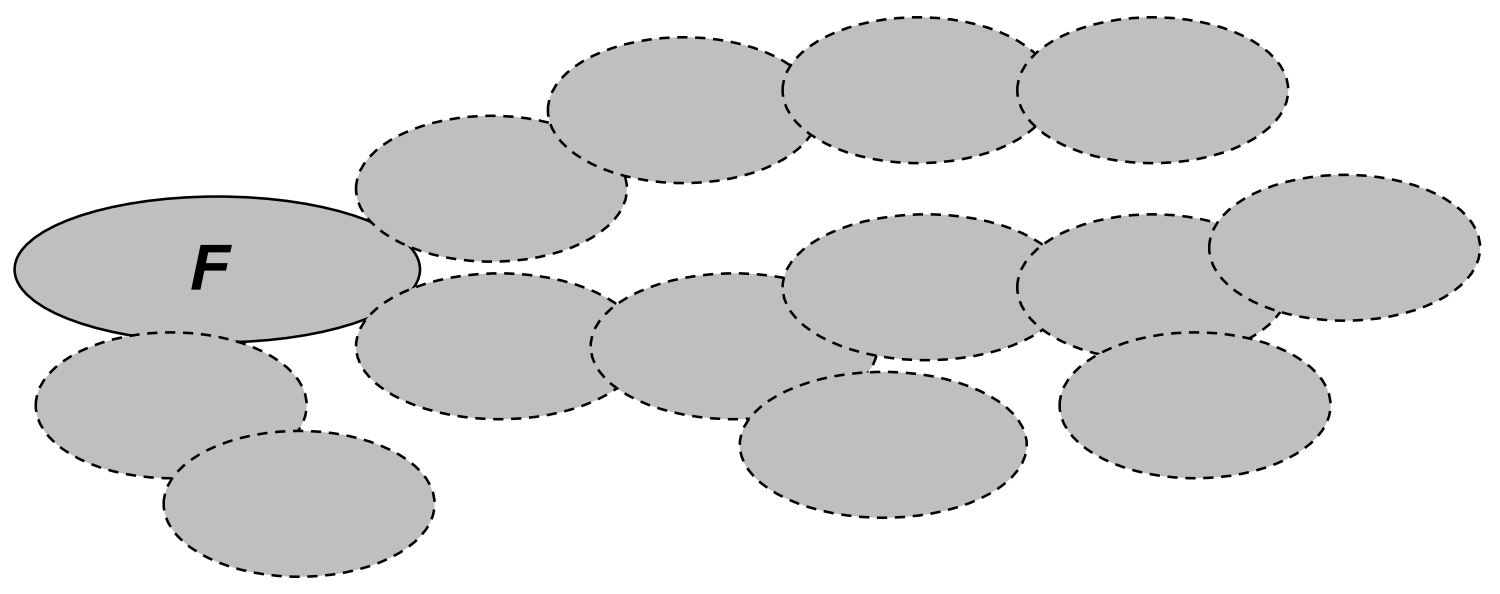

fes (finite saturation) is included in bts

(bound given by the number of terms in the finite " saturated fact »)

The decidability proof does not provide a halting algorithm (relies on the bounded treewidth model property [Courcelle 90]) 


\section{(Partial) Inclusion Map of Decidable Classes}

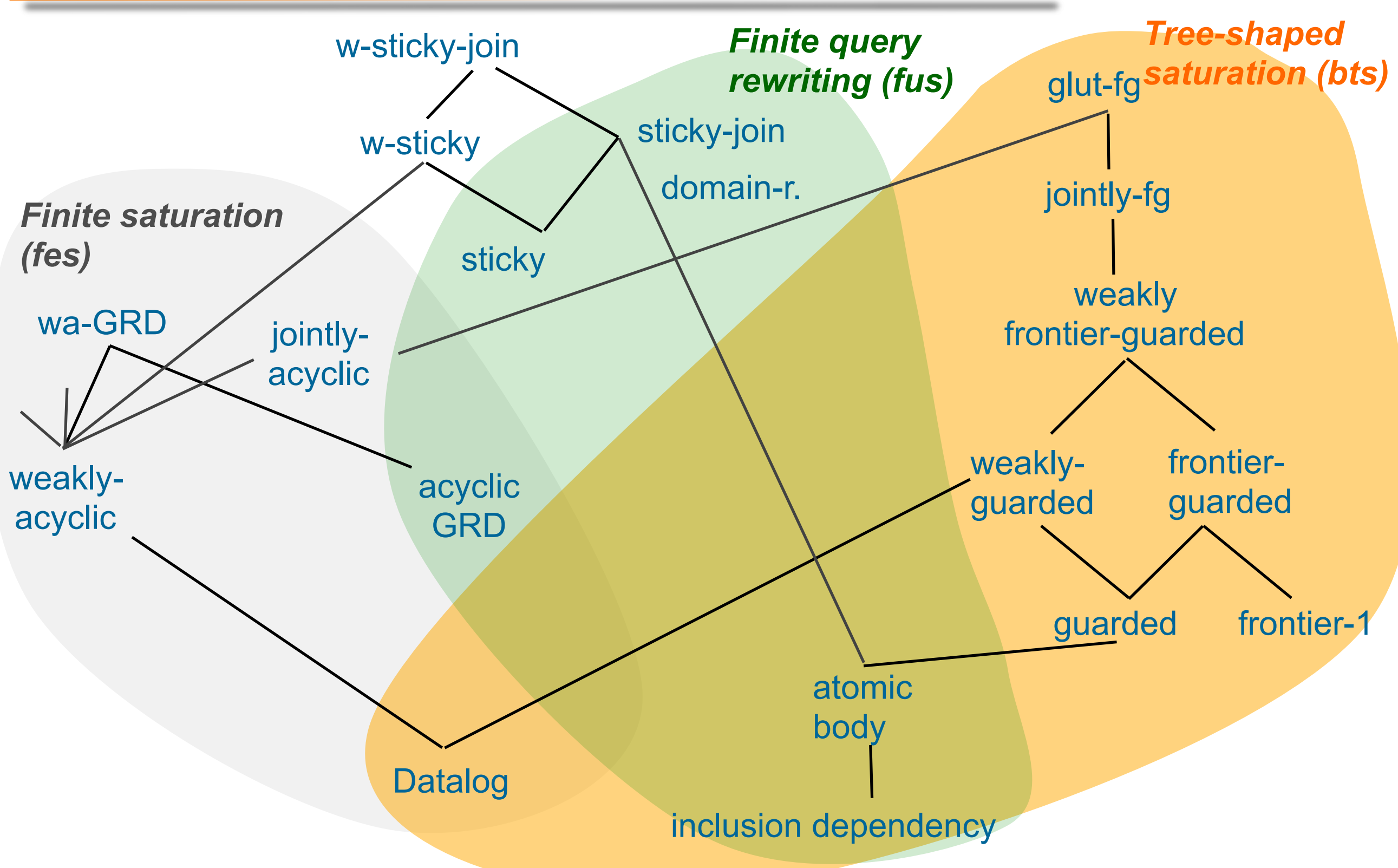




\section{(Partial) Inclusion Map of Decidable Classes}

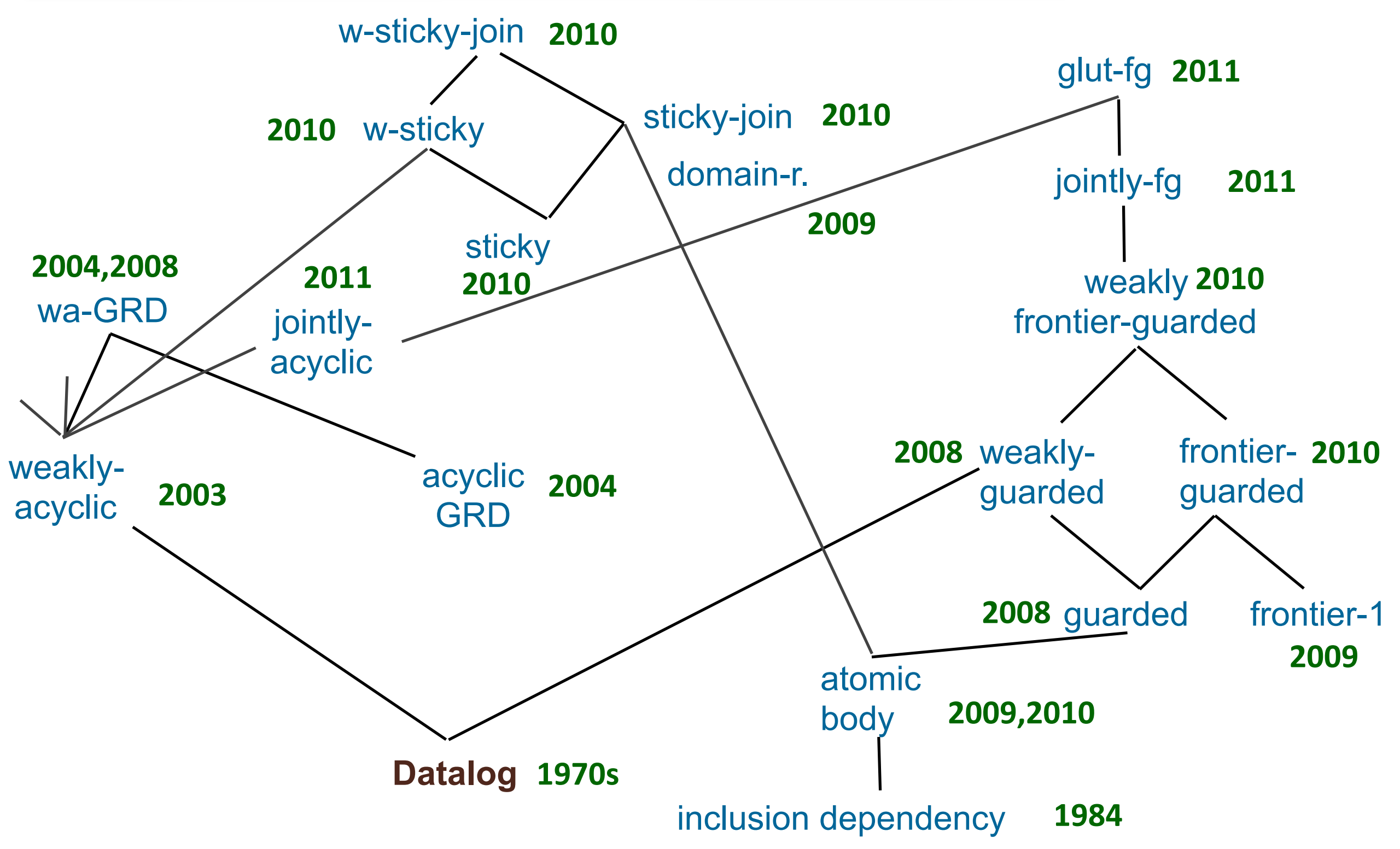




\section{Some Recognizable bts (and not fes) Classes of Rules}

Frontier: variables shared by the body and the head

Guard only the frontier

[Baget+ KR' 10] $r(x, y) \wedge r(y, z) \rightarrow$

$r(y, u) \wedge r(z, u)$

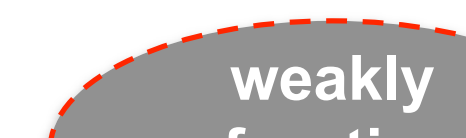

frontier

guarded

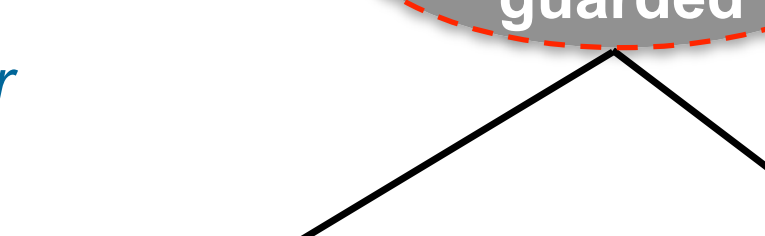

Guard only affected variables from the frontier

[Baget+ KR' 10]

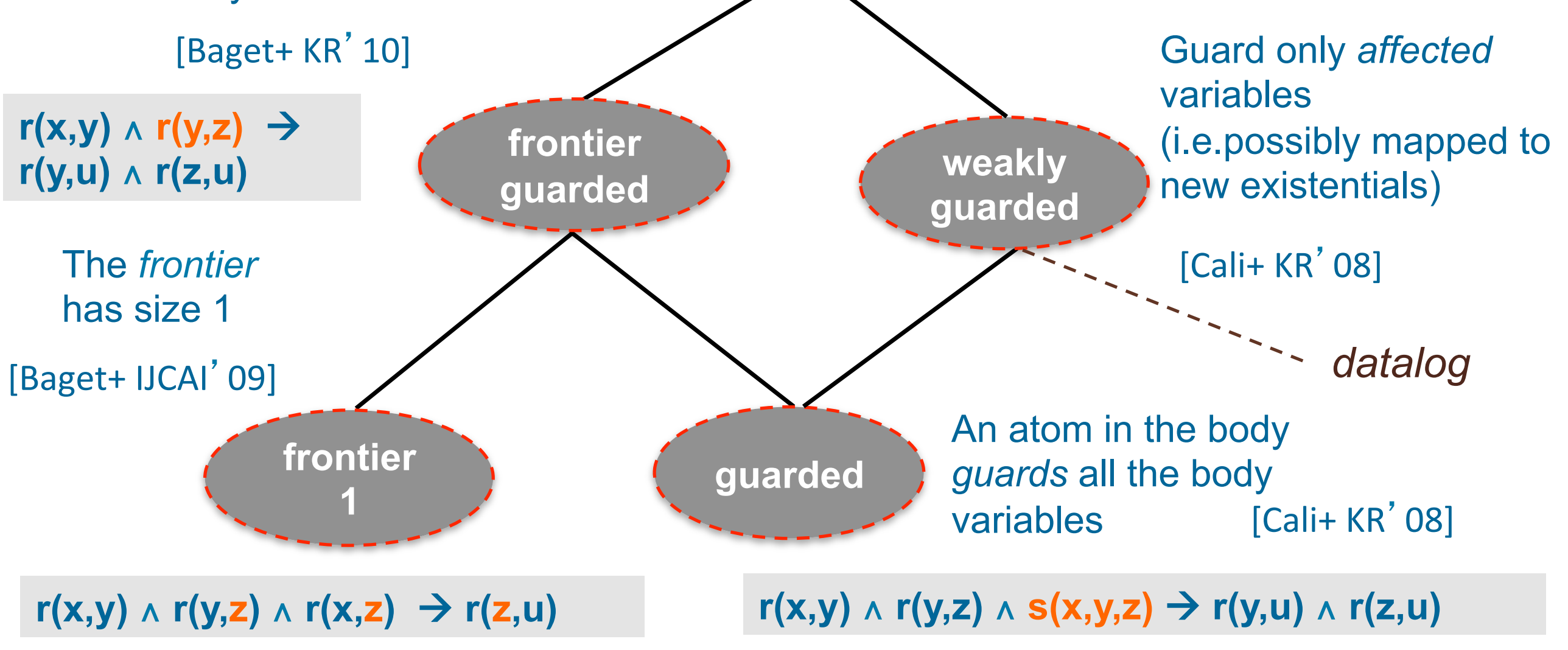

These classes are moreover « greedy bts » => a halting algorithm [Baget+ IJCAI" 11] 


\section{Greedy bts}

$$
\begin{aligned}
& R 1=p(x, y) \rightarrow p(y, z) \\
& R 2=p(x, y) \wedge q(x, z) \rightarrow r(x, y, t) \wedge p(y, t) \\
& F=p(a, b)
\end{aligned}
$$$$
q(b, z 0)
$$

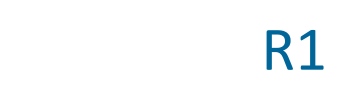

b z0

$$
p(a, b)
$$$$
a b
$$

$\mathrm{R} 2$
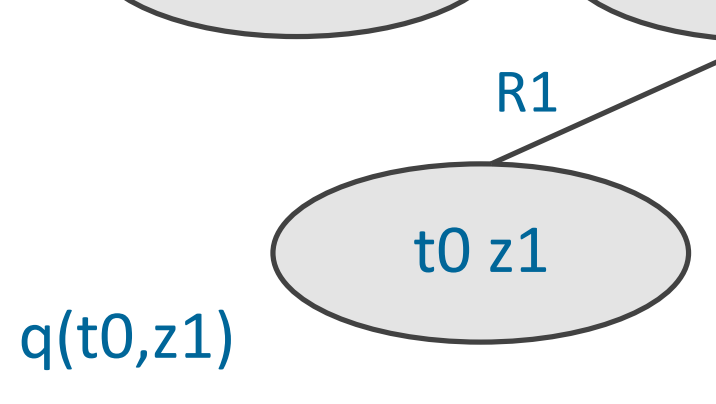

a b t0

$r(a, b, t 0)$ $p(b, t 0)$

Etc.

Greedy construction of a decomposition tree of the derived fact with bounded width 


\section{The « Greedy bts » Property [Baget+ IJCAI' 11]}

For any fact, for each rule application, frontier variables not being mapped to initial terms are jointly mapped to variables occurring in atoms added by a single previous rule application

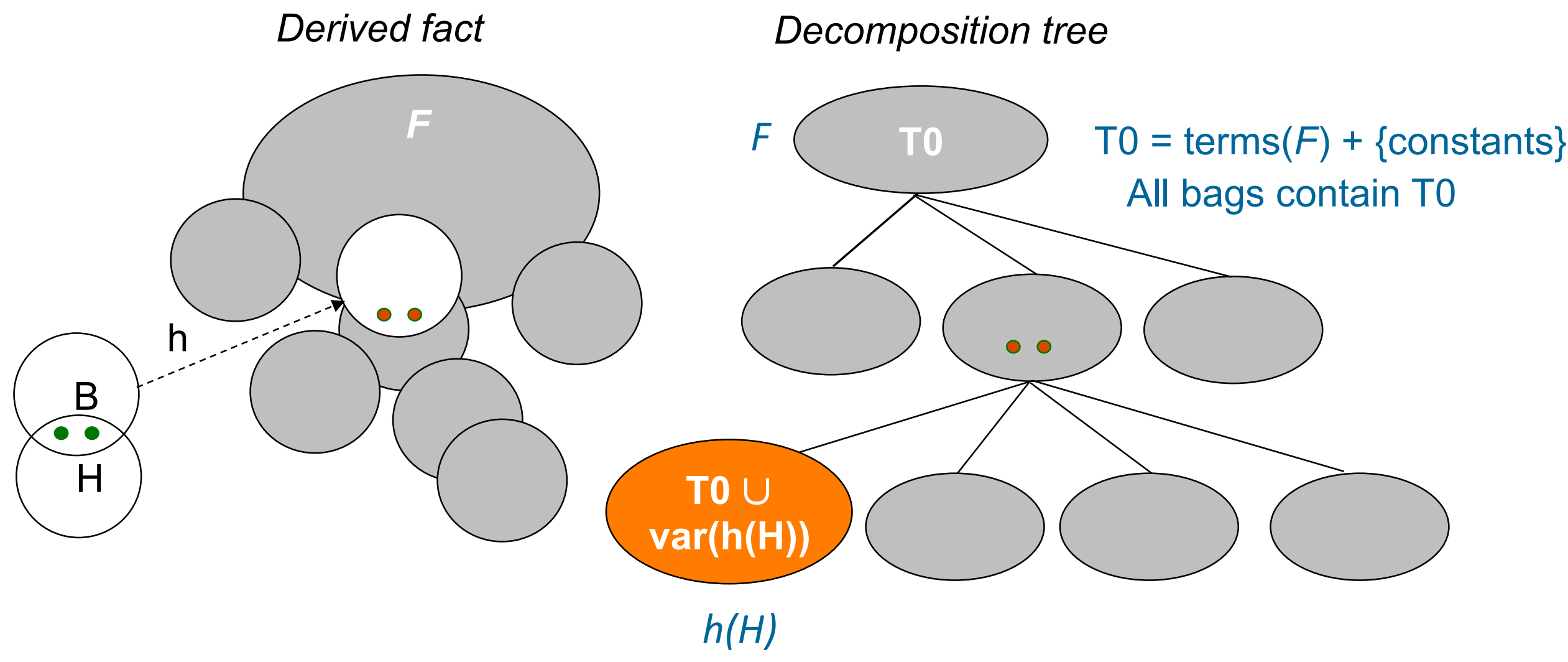




\section{Main Ideas of the Algorithm for gbts (1)}

Build a finite decomposition tree that encodes a potentially infinite fact

1. Bag pattern $=\{$ homomorphisms from part of a rule body to « current fact » that use some terms of the bag $\}$

$\rightarrow$ A rule is applicable to the current fact iff a bag pattern contains its body

$\rightarrow$ FC can be performed on the decorated tree

2. Equivalence relation on bags

Only one bag per equivalence class is developed The other nodes are blocked

Bounded number of equivalence classes $\rightarrow$ finite « full blocked tree » $T^{*}$ 


\section{Main Ideas of the Algorithm for gbts (2)}

\section{Query this finite decomposition tree}

[Baget+ IJCAI 2011] $Q$ seen as a rule « $Q \rightarrow$ match»

$Q$ is entailed iff it occurs in a bag pattern

i.e. $Q$ maps by homomorphism to atoms $\left(T^{*}\right)$

[Thomazo+ KR 2012] offline /online separation

(1) compilation: tree $T^{*}$ built independently from any query

(2) querying: any $Q$ is entailed iff it maps by *-homomorphism to $T^{*}$

i.e. $Q$ maps by homomorphism to a bounded « development » of $T^{*}$ 


\section{Backward Chaining: Unification Step}

$$
\begin{aligned}
& R=r(x) \rightarrow p(x, y) \\
& Q=p(u, v) \wedge p(u, w) \wedge p(v, w)
\end{aligned}
$$

Atomic unification:

$\mathrm{u} \rightarrow \mathrm{x} \quad \mathrm{v} \rightarrow \mathrm{y}$

$\mathrm{Q} 1=r(x) \wedge p(x, w) \wedge p(y, w)$

Soundness lost!

Indeed let $F=Q 1$

$\operatorname{saturation}(F, R) \cong F$

$Q$ does not map to $F$
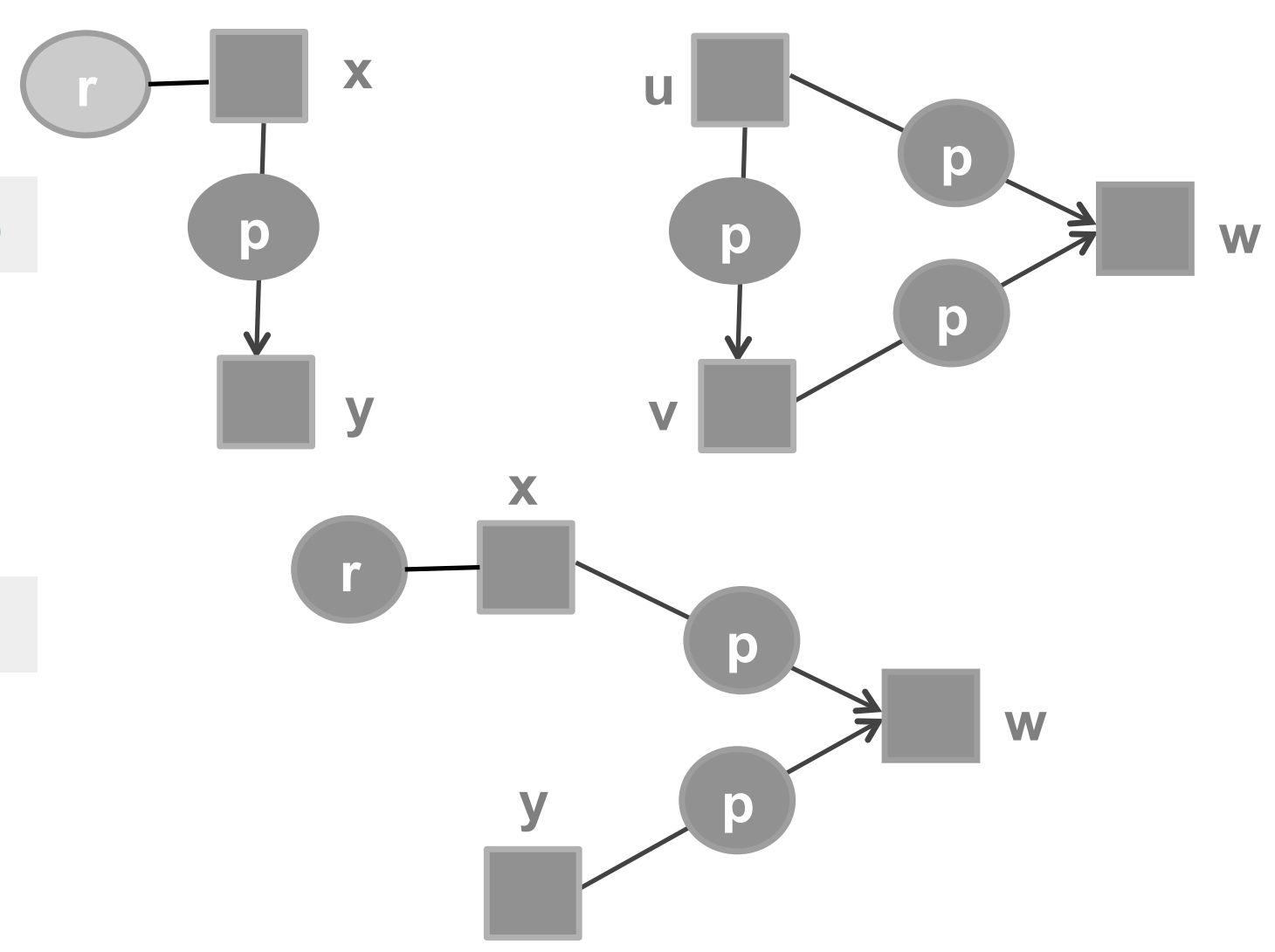

Existentials in rule heads produce a structure that must be taken into account 


\section{Key Notion: « Piece »}

- Given a subset $T$ of its variables, a set of atoms is partitioned into pieces.

A piece $=$ all atoms linked by $a$ « path » of variables not belonging to $T$

$$
\begin{aligned}
& p(u, v) p(v, w) p(w, u) s(u, u) \\
& T=\{u\} \\
& \text { Piece } 1=\{p(u, v) p(v, w) p(w, u)\} \\
& \text { Piece } 2=\{s(u, u)\}
\end{aligned}
$$

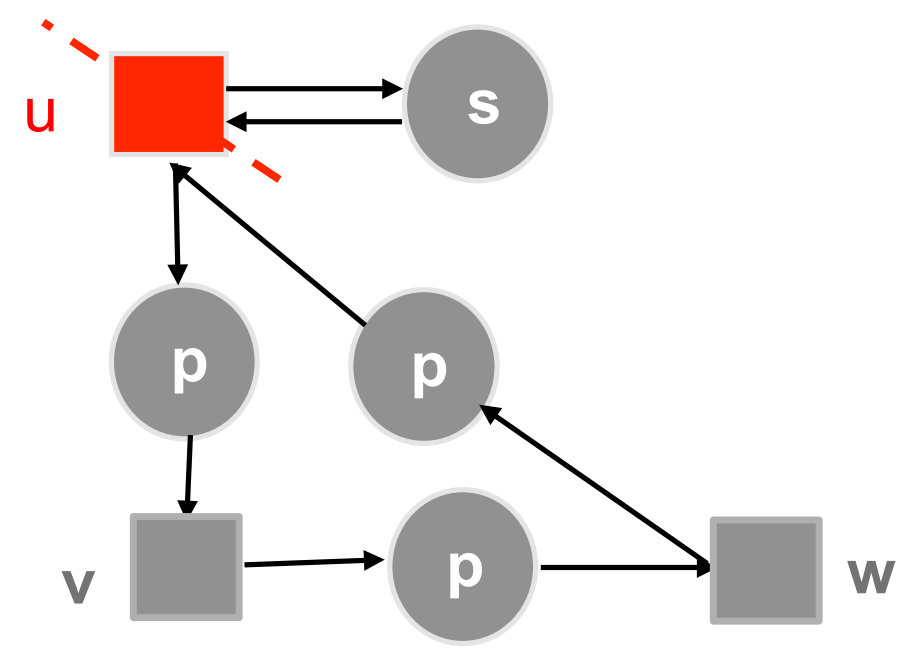

Basic notion for unification in backward chaining, dependency between rules, decomposition of a rule into equivalent rules, ... 


\section{Piece-Unification (1)}

$$
R=q(x, y) \wedge q(y, x) \rightarrow p(x, z) \wedge p(z, x) \wedge p(z, z) \wedge r(z) \quad Q=p(u, v) \wedge p(v, w) \wedge p(w, u) \wedge s(u, u)
$$
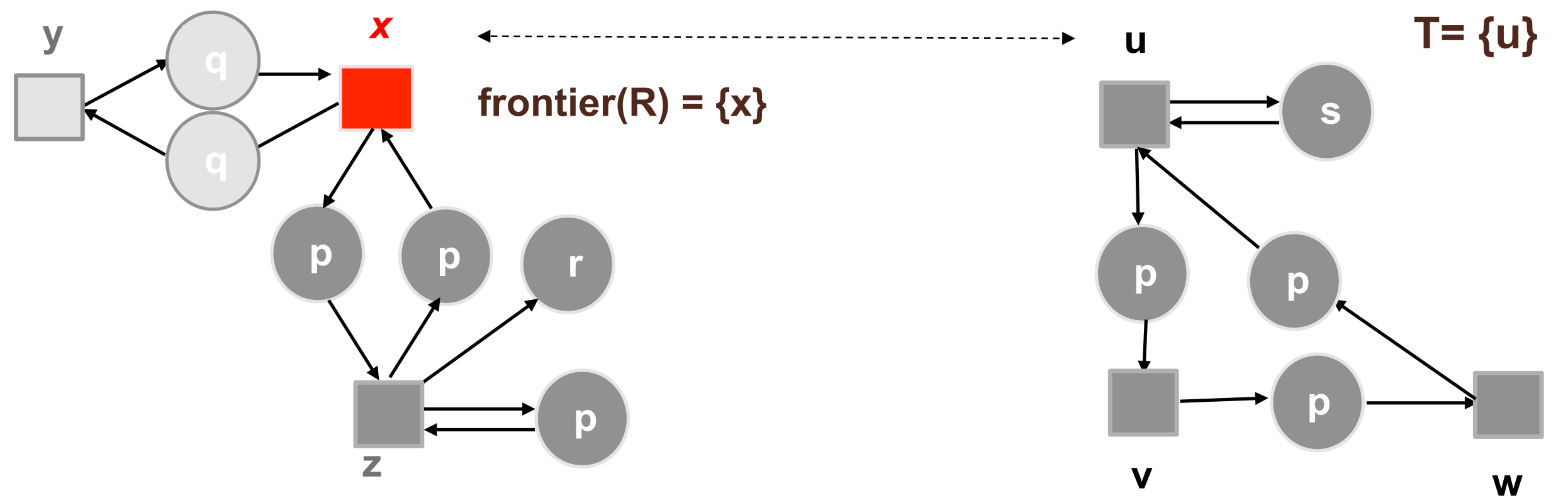

A piece-unifier has to map at least one piece of the query to the rule head

New query

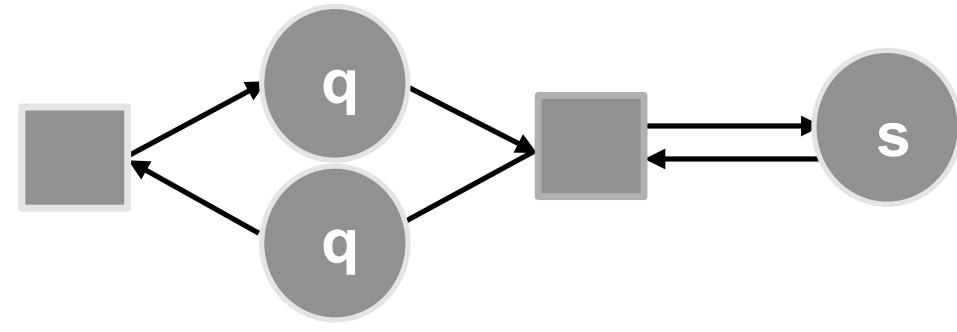




\section{Piece-Unification (2)}

$$
R=r(x) \rightarrow p(x, y) \quad Q=p(u, v) \wedge p(v, u)
$$
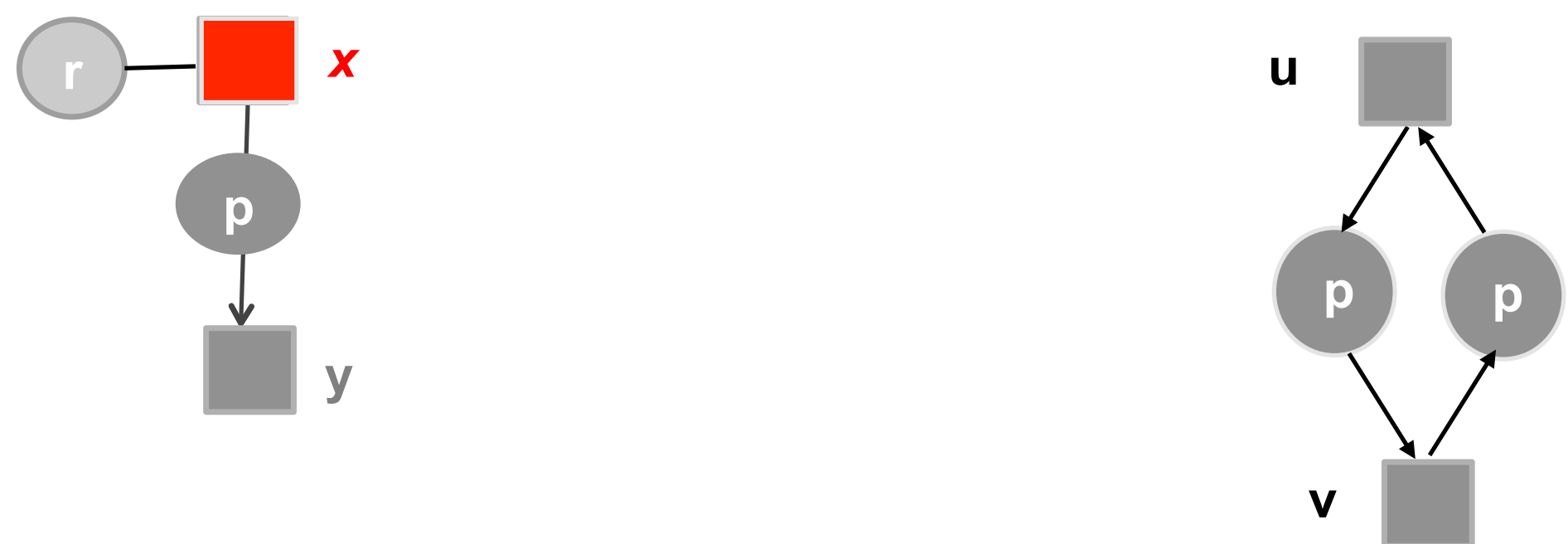

A piece-unifier has to map at least one piece of the query to the rule head

$\rightarrow$ failure 


\section{Piece-Unification (3)}

Initially [Salvat M... ICCS 1996] on conceptual graphs

Piece-unifier of a query $Q$ with a rule $R$ :

- a substitution $s$ of frontier $(R)$ by frontier $(R)+\operatorname{constants}(Q+h e a d(R))$

- a homomorphism $h$ from Q' $\subseteq$ Q to $s($ head $(R))$ s.t. $Q$ 'is a set of pieces according to $s$ and $h$

[Salvat M... 1996]

$F, R \mid=Q$ iff there is a sequence of piece-unifications that empties $Q$ (considering facts as rules with an empty body)

[Baget+ IJCAI 2009] for fus existential rules

$F, \mathcal{R} \mid=Q$ iff one of the piece-based rewritings of $Q$ maps to $F$ 


\section{Outline}

Existential rules: a logic- and graph-based framework

Decidability and algorithmic issues

- Focus on:

tree-shaped saturation in forward chaining piece-based unification in backward chaining

A (graph) tool for combining decidable classes of rules 


\section{Union of Decidable Sets of Rules}

- Next question:

is the union of two decidable sets of rules still decidable ?

practically:

can we safely merge several decidable ontologies ?

- can we build a decidable hybrid language from two languages whose semantics can be expressed by decidable subsets of rules?

- Bad news:

Almost all classes are pairwise incompatible

- Next question:

which conditions on the interactions between rules ensure compatibility? 


\section{A tool : the Graph of Rule Dependencies}

[Baget KR 2004, Baget+ IJCAI 2009, AlJ 2011]

$R 2$ depends on $R 1$ if applying $R 1$ may trigger a new application of $R 2$

i.e., there exists a fact $F$ s.t. $R 1$ is applicable to $F$ but $R 2$ is not and there is an application of $R 1$ to $F$ leading to $F^{\prime}$ s.t. $R 2$ is applicable to $F^{\prime}$

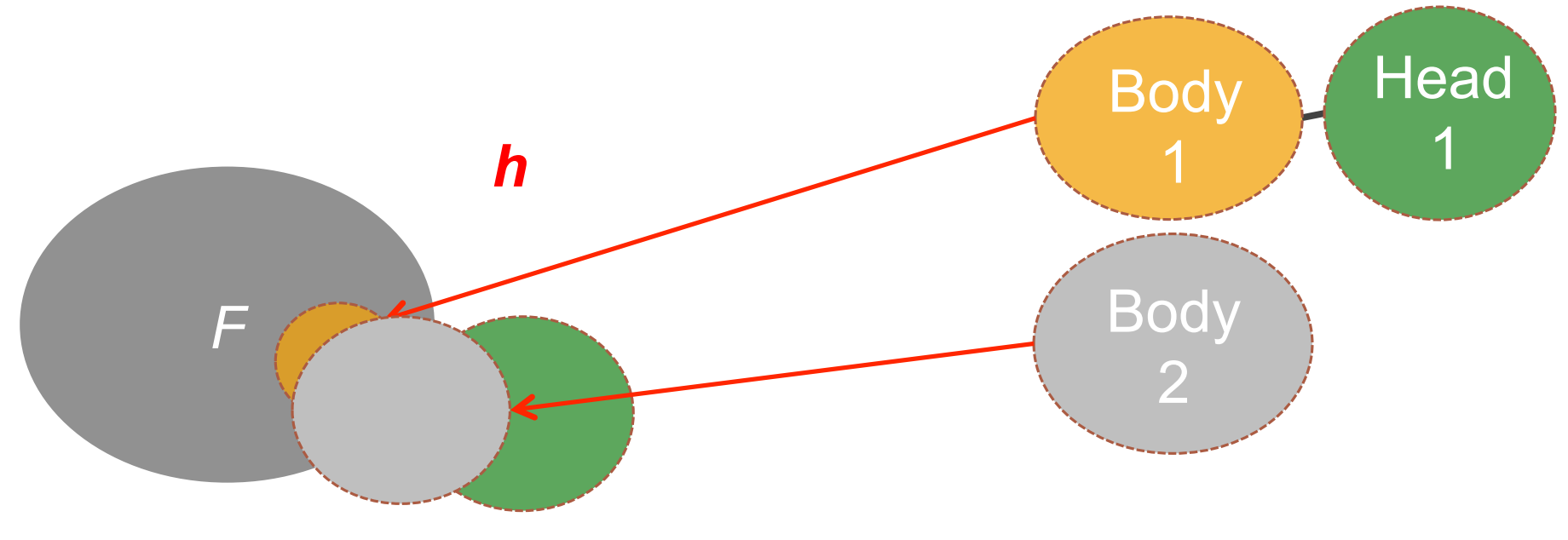

Effective computation of dependencies with a piece-unification test:

$\mathrm{R} 2$ depends on R1 iff there is a « piece-unifier » of body(R2) with head(R1) 
Combining Decidable Classes with the Graph of Rule Dependencies
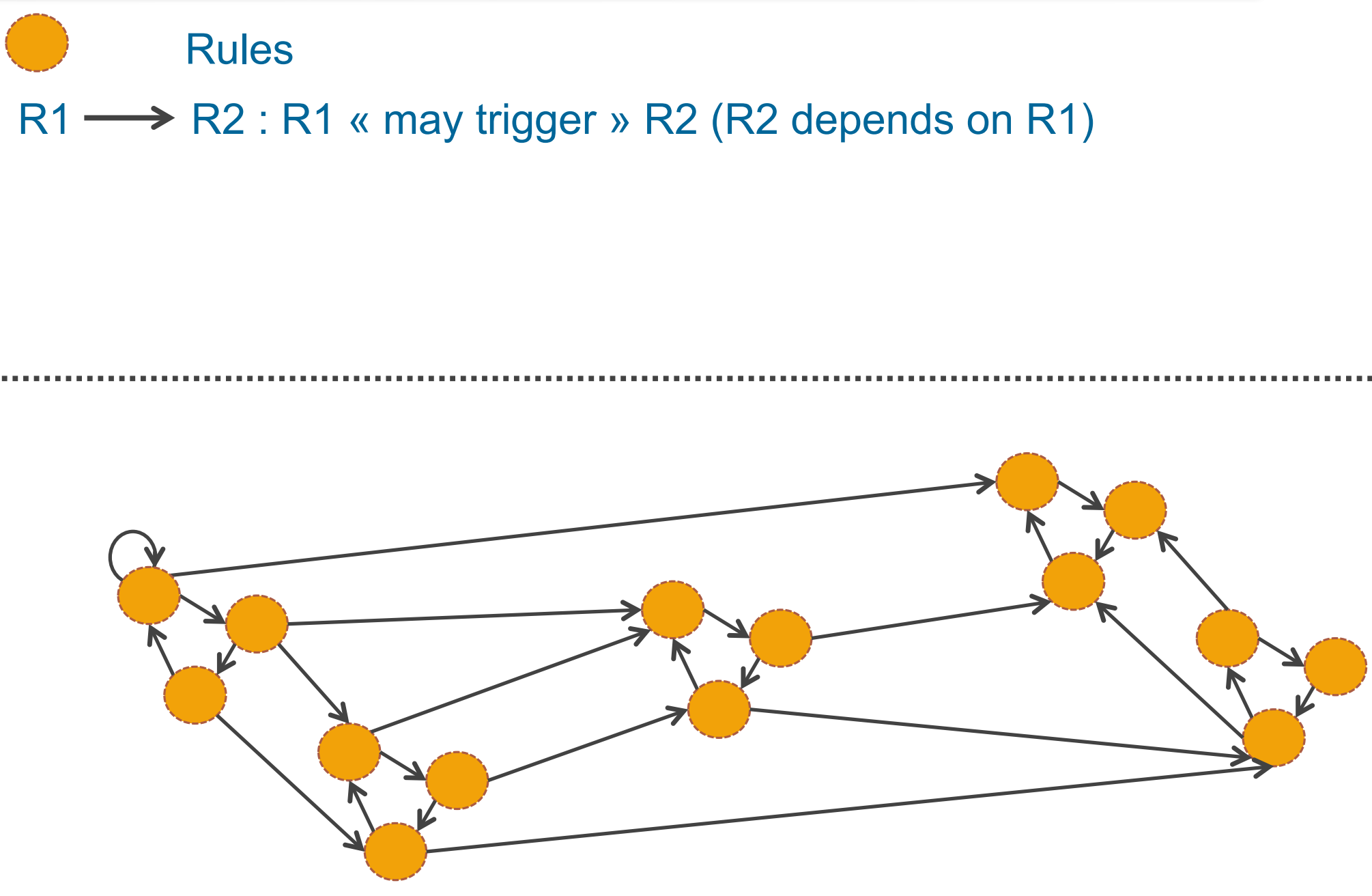
Combining Decidable Classes with the Graph of Rule Dependencies

If $\operatorname{GRD}(\mathcal{R})$ is without circuit then $\mathcal{R}$ is both fes (thus bts) and fus

fes $=$ finite fact saturation

fus $=$ finite query rewriting

$b t s=($ possibly infinite $)$ tree-shaped saturation

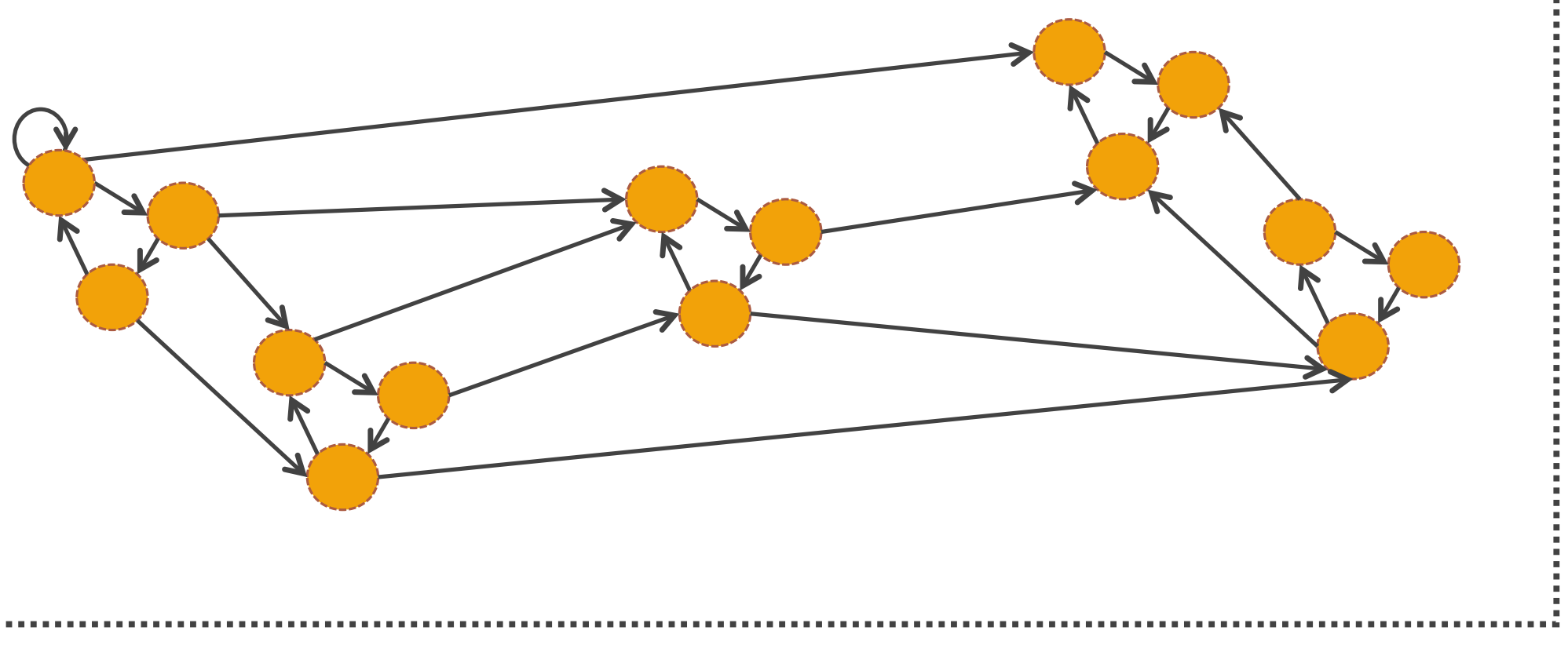


Combining Decidable Classes with the Graph of Rule Dependencies

\section{If all strongly connected components of $\operatorname{GRD}(\mathcal{R})$ are fes then $\mathcal{R}$ is fes [Baget 2004]}

The same holds for fus (but not for bts)

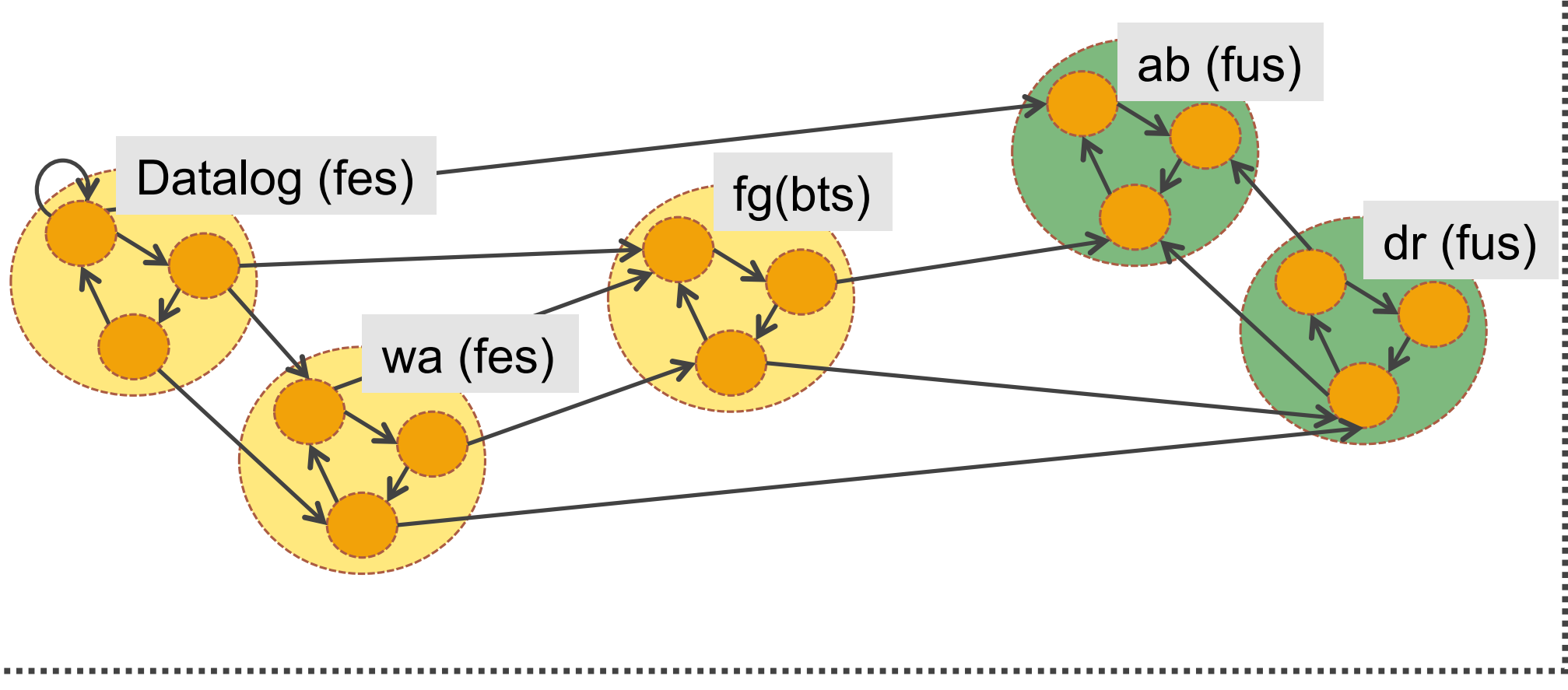


Combining Decidable Classes with the Graph of Rule Dependencies

\section{If all strongly connected components of $\operatorname{GRD}(\mathcal{R})$ are fes then $\mathcal{R}$ is fes [Baget 2004]}

The same holds for fus (but not for bts)

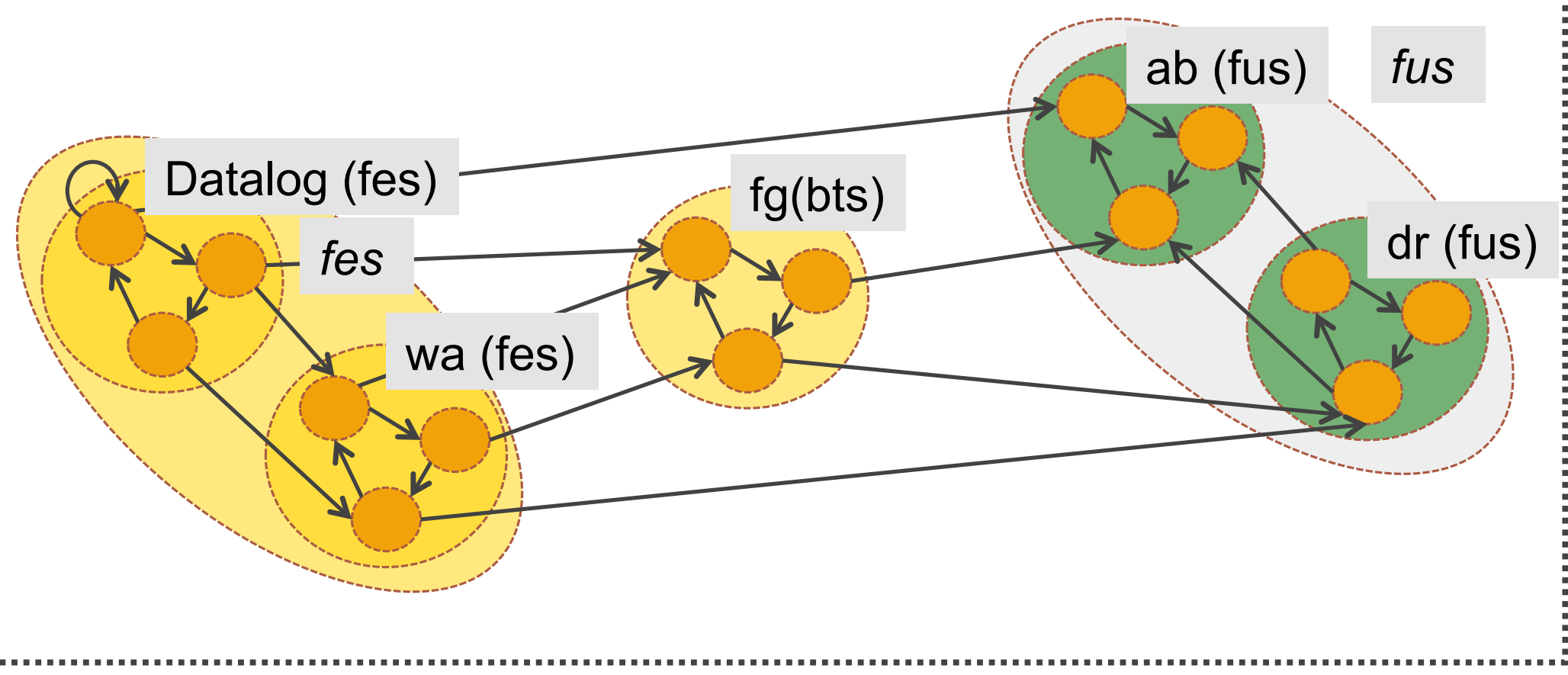


Combining Decidable Classes with the Graph of Rule Dependencies

Let $\mathcal{R}_{1}>\mathcal{R}_{2}$ be a partition of $\mathcal{R}$ s.t. no rule of $\mathcal{R}_{1}$ depends on a rule of $\mathcal{R}_{2}$

If $\mathcal{R}_{1}$ is fes and $\mathcal{R}_{2}$ is bts, then $\mathcal{R}$ is bts

If $\mathcal{R}_{1}$ is bts and $\mathcal{R}_{2}$ is fus, then $\mathcal{R}$ is decidable

\section{Decidable}

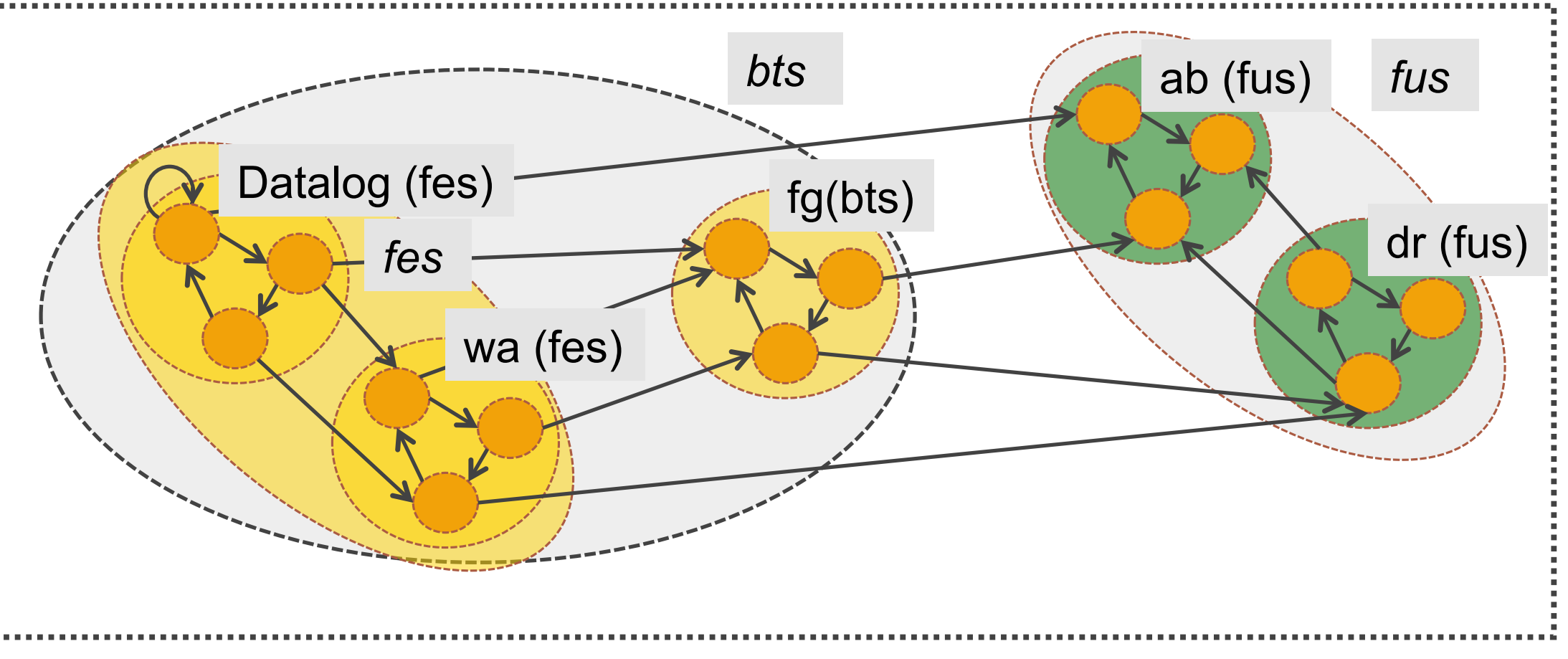




\section{Conclusion}

- An emerging rule-based framework for OBDA

- simple

- expressive

- flexible

Logic-based and Graph-based

Currently:

- A quite clear picture of decidable classes and their complexities

- First implementations - often for very specific subclasses

Next challenge: scalability 